\title{
An experiment on the adiabatic compressible turbulent boundary layer in adverse and favourable pressure gradients
}

\author{
By J. E. LEWIS, R. L. GRAN \\ TRW Systems Group, Redondo Beach, California \\ AND T. KUBOTA \\ California Institute of Technology, Pasadena, California
}

(Received 14 July 1971 and in revised form 20 September 1971)

A wind-tunnel model was developed to study the two-dimensional turbulent boundary layer in adverse and favourable pressure gradients without the effects of streamwise surface curvature. Experiments were performed at Mach 4 with an adiabatic wall, and mean flow measurements within the boundary layer were obtained. The data, when viewed in the velocity transformation suggested by Van Driest, show good general agreement with the composite boundary-layer profile developed for the low-speed turbulent boundary layer. Moreover, the pressure gradient parameter suggested by Alber \& Coats was found to correlate the data with low-speed results.

\section{Introduction}

There is a considerable body of experimental data dealing with the effect of both adverse and favourable pressure gradients on the low-speed turbulent boundary layer. The majority of the data taken prior to August 1968 has been meticulously analysed by Coles \& Hirst (1969). The result of this study is that with some notable exceptions (e.g. the experiments of Bradshaw \& Ferriss (1965) and Stratford (1959)) the data are well represented by experimentally defined 'laws', i.e. the law of the wall, and the velocity defect law. Both of these are contained within the composite boundary-layer profile used by Coles (1956):

$$
\frac{U}{U_{\tau}}=f\left(\frac{y U_{\tau}}{\nu}\right)+\frac{\tilde{\pi}}{\kappa} W\left(\frac{y}{\delta}\right)
$$

where $U_{\tau}=\left(\tau_{w} / \rho\right)^{\frac{1}{2}}$ is the friction velocity, $\nu$ is the kinematic viscosity, $\kappa$ is the von Kármán constant $=0.41, \tilde{\pi}$ is the wake strength, $W(y / \delta)$ is the wake function, and $\delta$ is the local boundary-layer thickness. Further, although the data show considerable scatter, the wake strength parameter $\tilde{\pi}$ appears to be reasonably correlated with Clauser's (1956) equilibrium pressure gradient parameter $\beta=\left(\delta^{*} / \tau_{w}\right)(d p / d x)$, indicating that most of the data represent a state of local equilibrium and are not strongly dependent on their upstream history.

The purpose of this paper is to describe an experimental investigation concerned with the effect of pressure gradient on the character and development 
of a compressible two-dimensional turbulent boundary layer formed on an adiabatic wall. It will be shown that the present data, when viewed in the velocity transformation suggested by Van Driest (1951), show good agreement with the classical wall and defect 'laws' with the exception of certain identified nonequilibrium profiles. The compressible pressure gradient parameter, suggested by Alber \& Coats (1969),

$$
\beta_{K}=\left(\delta_{K}^{*} / \tau_{w}\right)(d p / d x), \text { where } \delta_{K}^{*}=\int_{0}^{\delta}\left(1-U / U_{e}\right) d y
$$

( $\delta^{*}$ being the kinematic displacement thickness), is found to give the best correlation of the profiles with the low-speed results over the range of experimental data.

\section{Experimental procedure}

The major requirements which were defined for the experiment are as follows.

(i) The effects of compressibility must be identifiable.

(ii) The boundary layer must be effectively two-dimensional.

(iii) The effect of streamwise surface curvature must be eliminated.

(iv) The study must include the effects of both favourable and adverse pressure gradients.

(v) Redundant measurements must permit checking the self-consistency of the data.

The effects of compressibility are, of course, primarily a function of Mach number and hence the larger the Mach number, the greater the effects. Unfortunately, in existing continuous-flow facilities the maximum unit Reynolds number decreases as the Mach number increases; because of the necessity for a high Reynolds number for any turbulence experiment, a compromise was made and the experiment conducted at the intermediate Mach number of $4 \cdot 0$. The experiments were performed in the $40 \times 40 \mathrm{in}$. supersonic wind tunnel $A$ of the von Kármán Gas Dynamics Facility at the Arnold Engineering Development Centre, Arnold Air Force Station, Tennessee. The majority of the measurements were made at Mach 4 and at a free-stream unit Reynolds number of $0.50 \times 10^{6} \mathrm{in}^{-1}$.

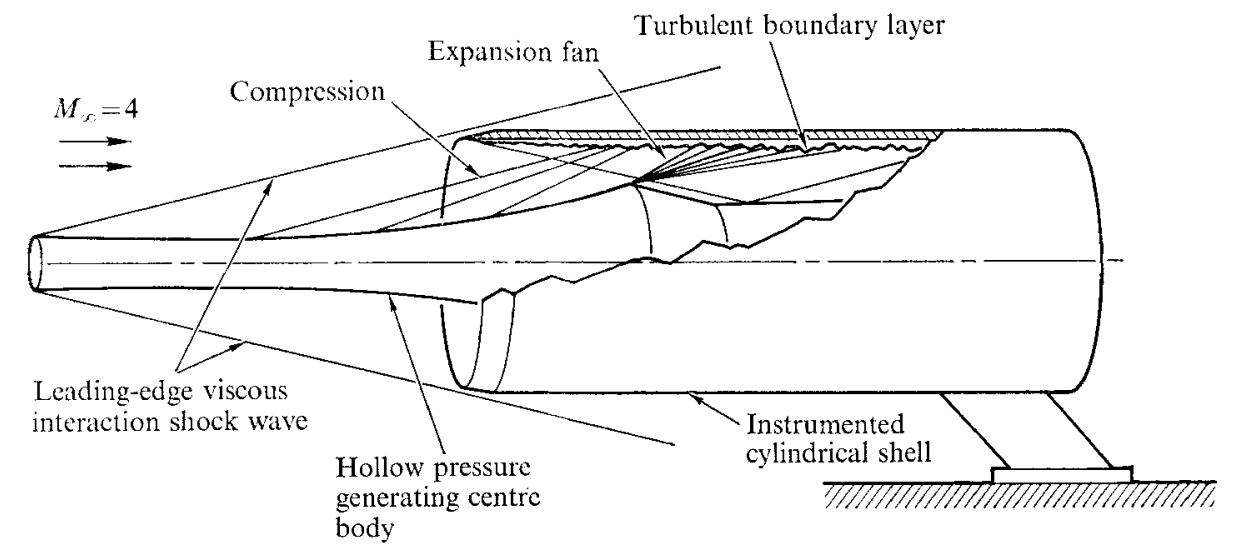

Figure 1. Wind-tunnel model. 
A wind-tunnel model (figure 1) was developed for the test which produced, as closely as is believed possible, an idealized two-dimensional turbulent boundary layer. The model consists basically of two parts: an outer shell and an inner pressure generating body. The outer shell is a hollow cylinder with an inside diameter of $20 \mathrm{in}$. and a length of $50 \mathrm{in}$. The boundary layer under study was formed on the inner surface of this outer shell. This geometry has several advantages. First, the problem of end effects associated with planar models of necessarily finite width is eliminated. Further, the large constant radius $\left(\delta / r_{w} \lesssim 0 \cdot 07\right)$ minimized the effects associated with axial symmetry and eliminated those associated with streamwise surface curvature. Finally, by utilizing different inner body configurations the boundary layer could be subjected to various pressure distributions.

The measurements consist of skin friction (Stanton tube measurements), wall temperature, wall pressure, Pitot pressure, total temperature and static pressure surveys across the boundary layer. Complete results are presented here for one configuration which resulted in the strongest adverse pressure gradients investigated. Constant pressure or 'flat plate' data are shown for overall comparison.

\section{Data reduction procedure}

The Pitot pressure data and the measured wall pressure at a given survey location were used to calculate the Mach number profile $M(y)$ across the boundary layer. Static pressure measurements and the edge Pitot pressure were used to verify that the static pressure was constant (within $\pm 10 \%$ ) across the boundary layer. The velocity profile was then computed from $M(y)$ and the total temperature measurements, $T_{T}(y)$. These latter measurements, although they had little effect on the resultant velocity profile since $\left(T_{T e}-T_{w}\right) / T_{T e} \approx 0.08$, did appear to extrapolate to the wall temperature and, within experimental accuracy, verified the Crocco relation

$$
\left(T_{r}-T_{w}\right) /\left(T_{T e}-T_{w}\right)=U / U_{e}
$$

In the above, $T_{w}$ is the wall temperature and the subscript $e$ refers to values at the edge of the boundary layer.

The integral properties at each survey station (e.g. momentum thickness $\theta$ ) have all been evaluated in a straightforward manner by using the experimental data, with the exception of the contribution to these integrals from the sublayer. This has been included by using Coles's (1953) tabulated sublayer function for the region $0<y U_{\tau} / v_{w}<50$ and is expressed as

$$
U^{*} / U_{\tau}=f\left(y U_{\tau} / \nu_{w}\right)
$$

The evaluation of $U_{\tau}$ along with the relation between the transformed velocity $U^{*}$ and the physical velocity $U$ is described in the next section. The contribution of the sublayer to the integral properties was generally quite small, but the authors considered the use of a sublayer function to be more consistent than truncating or otherwise approximating the integrals.

In order to evaluate the local pressure gradient $d p / d x$, necessary for certain comparisons to be made, the authors selected analytical expressions for the edge Mach number $M_{e}$, based on the measured data. An example of such an expression 
is shown in figure 2 along with the measured edge Mach number computed by two different methods. This procedure is satisfactory except in the neighbourhood of $x=13.5 \mathrm{in}$. and $x=18.5 \mathrm{in}$., where the selected curve fit predicts a discontinuous slope.

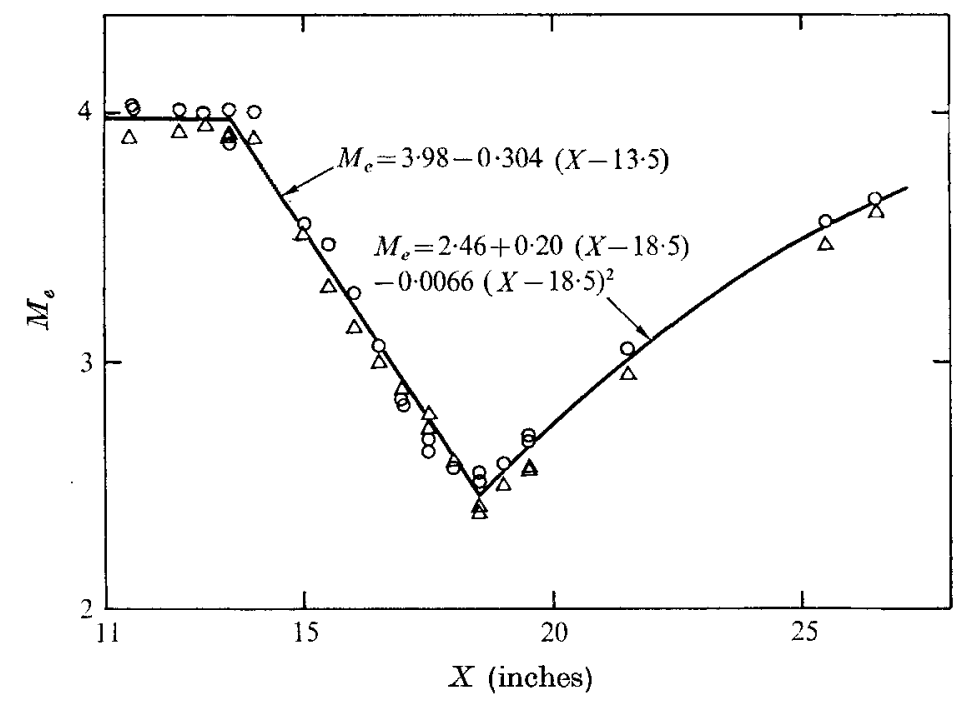

Figure 2. Edge Mach number distribution. O, calculated using Pitot-isentropic relation; $\triangle$, calculated using Pitot and wall pressure.

\section{Experimental results}

\subsection{Profile interpretation}

Quite early in the analysis of the data it became clear that, in order to extract the maximum available information, some method which could distinguish the effects of pressure gradient from the concurrent effects of varying Mach number and Reynolds number would have to be devised. The results of what are generally classified as transformations of the compressible flow equations to an equivalent low-speed form (e.g. the work of Baronti \& Libby (1966) and the general approach taken by Coles (1962) along with the extension to non-constant pressure flows by Lewis, Kubota \& Webb (1970)) have not been entirely successful in delineating the effects of Mach number and/or pressure gradient. Using the formulation of Van Driest (1951), Maise \& MeDonald (1967) were able to show that the effects of compressibility were well described for the special case of the adiabatic flat plate boundary layer; this formulation was followed in analysing the present data.

The first attempts at applying this approach to the data were encouraging although some inconsistencies were noted. Since the original skin friction estimates were made using Stanton tubes, it was not clear whether these inconsistencies were caused by an inadequate velocity transformation or by the skin friction measurements.

In order to test the validity of the velocity transformation, the authors adopted an approach similar to that developed by Coles \& Hirst (1969). Basically, this 
involves invoking a composite velocity profile assuming that the effects of compressibility are suitably accounted for through Van Driest's transformation. This is expressed as

$$
\frac{U^{*}}{U_{\tau}}=f\left(\frac{U_{\tau} y}{\nu_{w}}\right)+\frac{\tilde{\pi}}{\kappa} W\left(\frac{y}{\delta}\right)
$$

where $U^{*}$ is the Van Driest velocity transformation given by

$$
\frac{U^{*}}{U_{e}}=\frac{1}{A}\left\{\sin ^{-1}\left(\frac{2 A^{2}\left(U / U_{e}\right)-B}{\left(B^{2}+4 A^{2}\right)^{\frac{1}{2}}}\right)+\sin ^{-1}\left(\frac{B}{\left(B^{2}+4 A^{2}\right)^{\frac{1}{2}}}\right)\right\}
$$

with

$$
A^{2}=\left(T_{e} / T_{w}\right) \times \frac{1}{2}(\gamma-1) M_{e}^{2}, \quad B=T_{e} / T_{w}+A^{2}-1
$$

The function $f$ appearing in (4) was taken as $(1 / \kappa) \ln \left(y U_{\tau} / \nu_{w}\right)+C(\kappa=0.41$ and $C=5 \cdot 0$ ) for $y U_{\tau} / \nu_{w}>50$. The 'wake function' $W(y / \delta)$ was taken as $2 \sin ^{2}(\pi y / 2 \delta)$, exactly as in the low-speed case.

The above equations were then compared with the experimental profile data and the two parameters $U_{\tau} / U_{e}$ and $\delta$ varied until a 'best' fit (in the leastmean-squares sense) was determined. The remaining parameter, $\tilde{\pi}$, appearing in (4) is not independent since at the outer edge of the boundary layer $y=\delta$ and $U=U_{e}$ and thus,

$$
\frac{2 \tilde{\pi}}{\kappa}=\frac{U_{e}^{*}}{U_{\tau}}-\frac{1}{\kappa} \ln \frac{\delta U_{\tau}}{\nu_{w}}-C .
$$

In performing this fitting operation, the authors followed the procedure given by Coles \& Hirst. For example, data points very close to the wall were not used, by limiting the fitting region to $y>50 \nu_{w} / U_{\tau}$ on the grounds that the function is correct but the data is not. Similarly, points near the outer edge of the boundary layer were also excluded on the basis that the data is correct but that the function is not. However, unlike Coles, who chose to select the value of $y / \delta$ above which data were excluded (usually taken as 0.9 but varied depending upon the circumstances to 0.75 or less), we have chosen to exclude those data points for which $U / U_{e} \geqslant 0.98$. This approximation accomplished much the same end results but involves a less subjective bias.

\subsection{General results}

In order to justify the procedure described above, the values of wall-shear stress thus obtained were compared to the Stanton tube measurements in figure 3 (where $\Delta p \equiv p_{s t}-p_{w}$ and $h$ is the Stanton tube height) along with the results of a number of previous Stanton tube calibrations. The values for wall shear stress inferred from the velocity profiles are seen to be consistent with the Stanton tube measurements. Furthermore, the skin friction coefficients inferred from the 'flat plate' profiles deviated by less than 5\% from a theoretical prediction $\uparrow$ at the same Mach number and Reynolds number based on the momentum thickness.

$\dagger$ This theoretical prediction was taken from Hopkins \& Inouye (1971) and uses the 'Van Driest II' formulation, which appears to give the best agreement with previous flat plate compressible data. It should be pointed out that this formulation uses a different mixing length expression from the velocity transformation described herein. However, no inconsistency results since this theory has been used solely for computing the flat plate skin friction. 


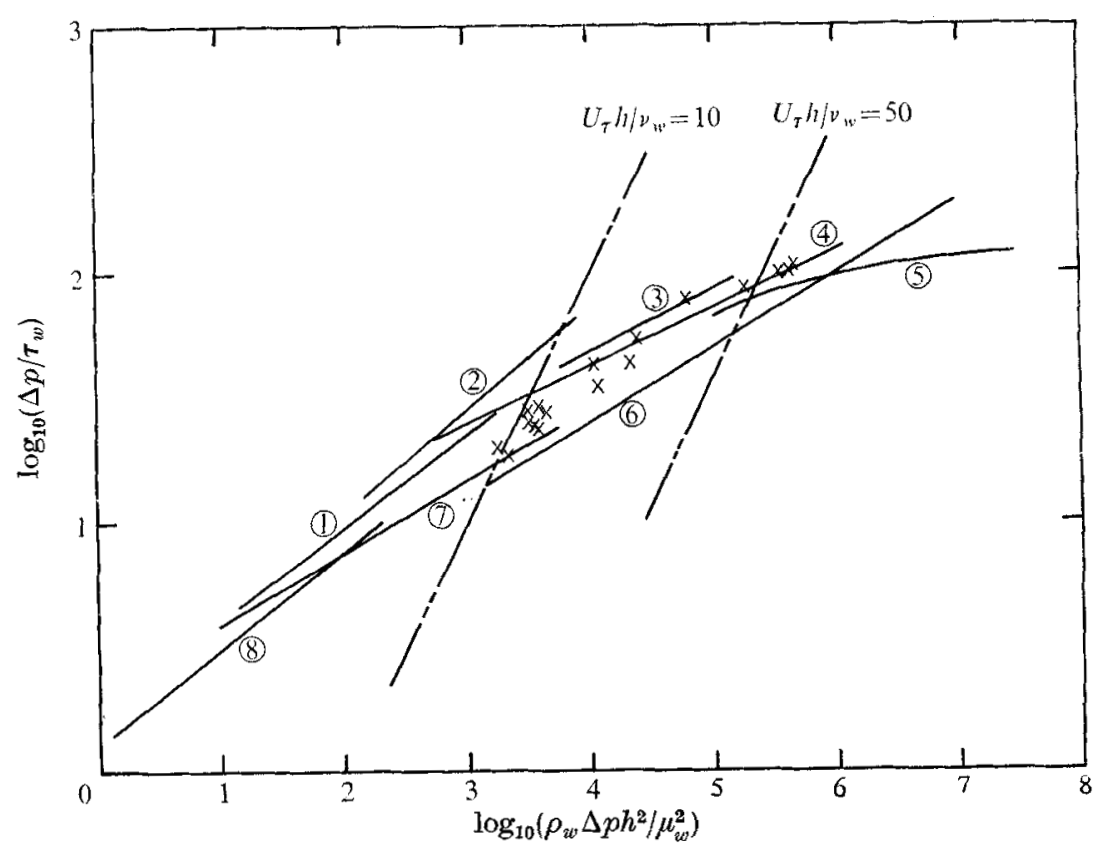

FIGURE 3. Stanton tube measurements using velocity profile inferred shear stress. $x$, present results; (1), Hool (1956), laminar duct flow; (2), Abarbanel et al. (1959), Preston tube, subsonic and laminar supersonic flow; (3), Abarbanel et al. (1959), Preston tube, supersonic turbulent flow; (4) Smith et al. (1964), turbulent supersonic; (5), Hopkins \& Keener (1966), turbulent supersonic flow; (6), Bradshaw \& Gregory (1961), subsonic turbulent; (7), Brott et al. (1968), turbulent supersonic, adiabatic and cold wall.

The calculated values of $\tilde{\pi}$ for the 'flat plate' data also give evidence in favour of the fitting procedure described. All of these values were in the range

$$
0 \cdot 5 \lesssim \tilde{\pi} \lesssim 0 \cdot 58
$$

which is virtually the same as the range found for incompressible flows. This result should be contrasted with the result of the aforementioned transformation theories (e.g. Baronti \& Libby 1966) which indicate that the 'flat plate' value of $\pi$ is a strong function of Mach number.

The development of the boundary layer when subjected to the edge Mach number distribution of figure 2 is shown in figure 4 . It is evident that the development of the quantities $\delta, R e_{\theta}$ and $C_{f e}$ is significantly enhanced or diminished by the adverse pressure gradient $(13.5 \mathrm{in} .<x<18.5 \mathrm{in}$.) and the favourable pressure gradient $(18.5 \mathrm{in} .<x<26.5 \mathrm{in}$.). It should be pointed out that $\delta$, as shown here, is the value obtained from the aforementioned reduction procedure as opposed to a commonly made choice corresponding to $U / U_{e}=0 \cdot 995$. This latter definition gives values about $10 \%$ lower than those indicated.

The values of the wake strength $\tilde{\pi}$ and the pressure gradient parameter $\beta_{K}=\left(\delta_{K}^{*} / \tau_{w}\right)(d p / d x)$ are shown in figures $5(a)$ and $(b)$, respectively. Other possible parameters describing the 'strength' of the pressure gradient (such as 


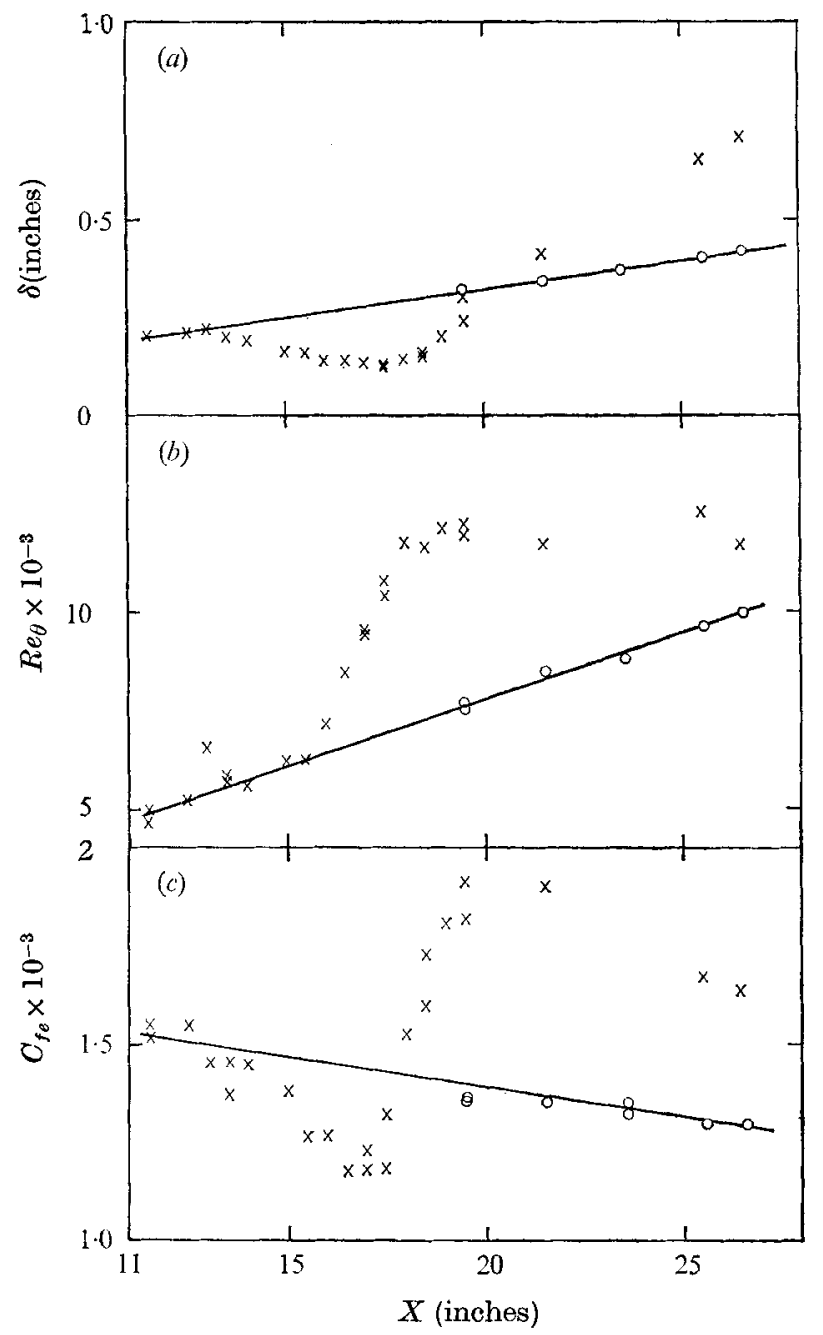

Figure 4. The development of the boundary layer when subjected to the edge Mach number distribution of figure 2, - , flat plate. (a) Boundary-layer thickness. (b) Local Reynolds number based on momentum thickness. (c) Local skin friction coefficient.

$\left.\left(\theta / M_{e}\right) d M_{e} / d x\right)$ were considered, but none were found to exhibit as good a correspondence with $\tilde{\pi}$ as $\beta_{K}$ or $\beta$. (The pressure gradient parameter $\beta=\left(\delta^{*} / \tau_{w}\right) d p / d x$ is about twice as large as $\beta_{K}$.)

The results of the boundary-layer momentum balance are shown in figure 6 . The left- and right-hand sides of the integrated momentum equation

$$
\frac{q_{e} \theta}{\left(q_{e} \theta\right)_{0}}-1-\frac{1}{2} \int_{p_{0}}^{p} \frac{\delta^{*}}{\theta_{0}} \frac{d p}{q_{e_{0}}}=\frac{1}{2} \int_{x_{0}}^{x} \frac{\tau_{w}}{q_{e_{0}}} \frac{d x}{\theta_{0}},
$$

where $q_{e}=\frac{1}{2} \rho_{e} u_{e}^{2}$, are plotted in this figure as the abscissa and the ordinate respectively. Perfect agreement between these is indicated by the solid line, while error bounds of $\pm 15 \%$ are indicated by the dashed lines. The close experimental 
agreement between these quantities suggests that the desired two-dimensionality of the boundary layer and the overall consistency of the data have been achieved.

The Van Driest velocity profiles for selected stations are shown in figure $7(a)$ for the adverse pressure gradient and in figure $7(b)$ for the favourable pressure gradient. Also indicated by a solid line is the logarithmic law of the wall,

$$
U^{*} / U_{\tau}=(1 / \kappa) \ln \left(y U_{\tau} / \nu_{w}\right)+\tilde{5} \cdot 0
$$

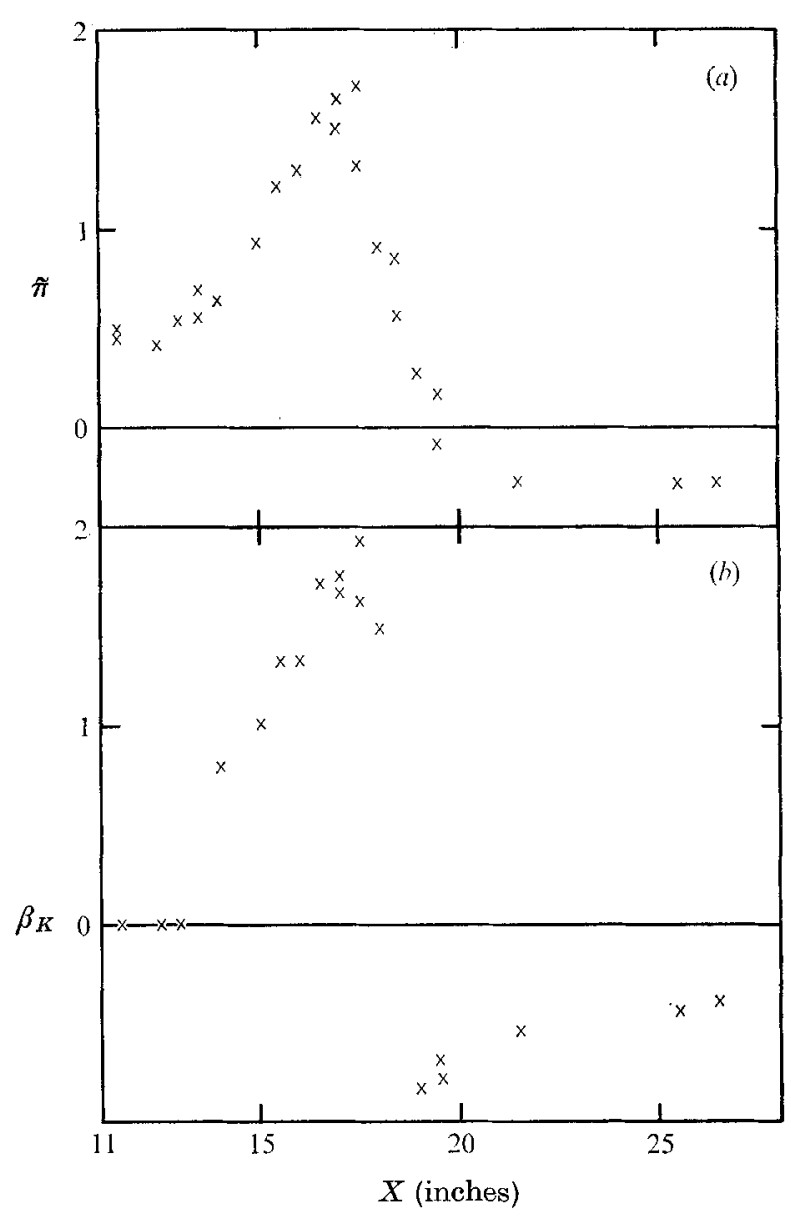

Figure 5. (a) Wake strength parameter as a function of $X$.

(b) Pressure gradient parameter as a function of $X$.

The agreement between the data and the law of the wall is seen to be quite good. The wake functions $W(y / \delta)$ for these profiles are shown in figures $8(a)$ and $8(b)$ for the adverse and favourable pressure gradients respectively. Also shown is the commonly accepted analytical form of the wake function mentioned previously. Note the excellent correspondence between the data and the functional form for the adverse pressure gradient region. On the other hand, the favourable pressure gradient region does show substantial deviations from the accepted form which will be discussed later. 


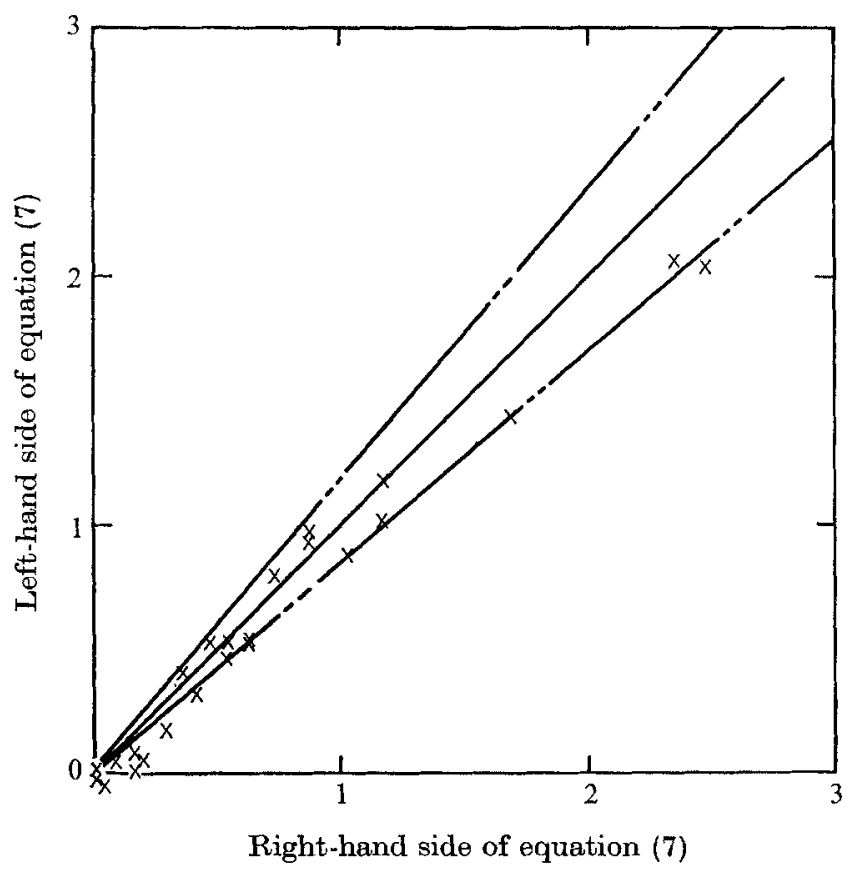

Figure 6. Momentum balance. —, line of perfect agreement; $-\cdots- \pm 15 \%$ error limits; $x$, present results.

\section{Correlation with low-speed data}

\subsection{Adverse pressure gradient}

A normalized local skin friction coefficient is plotted against the pressure gradient parameter $\beta_{K}$ in figure 9 . Here, an attempt has been made to eliminate the dependence on Mach number and Reynolds number by normalizing with the theoretical flat plate value corresponding to the same value of $M_{e}$ and $R e_{\theta}$. This theoretical value was computed using the procedure given in Hopkins \& Inouye (1971). Also shown is similarly normalized low-speed data given by Coles \& Hirst. As can be seen, the comparison between the high-speed and the low-speed data is quite good for $\beta_{K} \geqslant 0$.

The values of $\#$ have been plotted against $\beta_{K}$ in figure $10, \uparrow$ again including low-speed data. The low-speed data exhibit large scatter but nevertheless a general trend is apparent. The high-speed data exhibit the same trend for the adverse pressure region and moreover are well within the low-speed scatter. (In view of the fact that $\beta$ is roughly twice as large as $\beta_{K}$, the use of $\beta$ would not give as good a correlation with the low-speed data.)

$\dagger$ The correlations for both $\pi$ and $G$ (figure 11) would be even better if the estimate of the pressure gradient at $x=13$ and $18 \mathrm{in}$. were reduced. While the authors feel that their true values are indeed lower than that derived from the analytic expression, this modification has been omitted. 


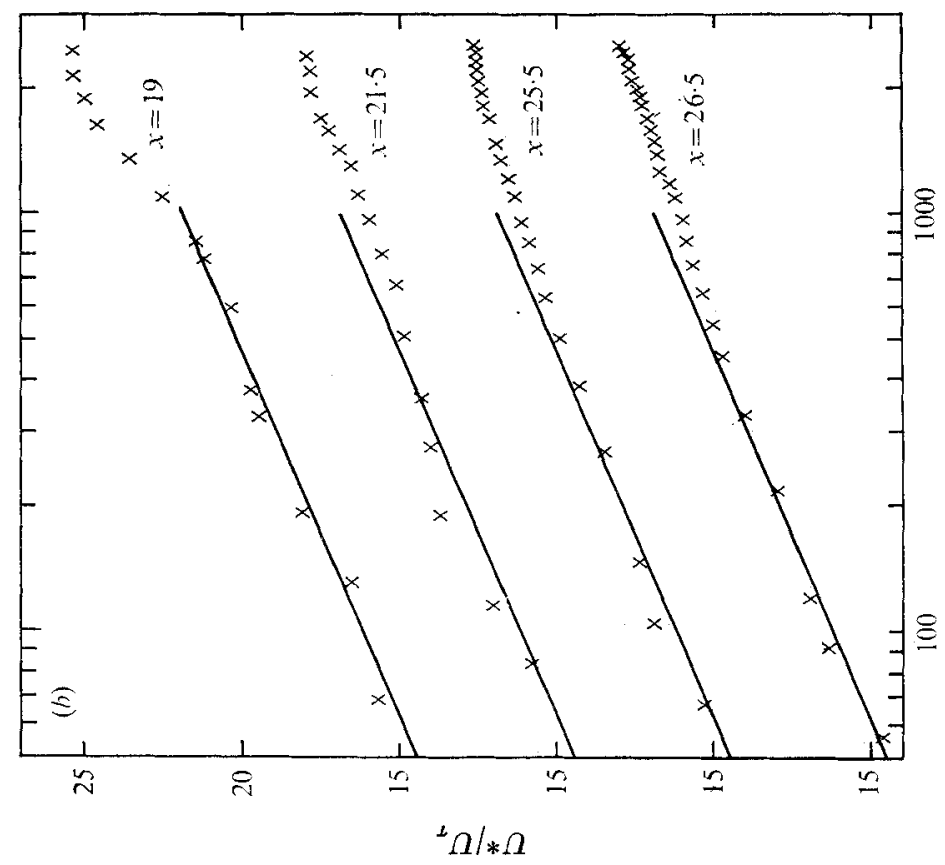

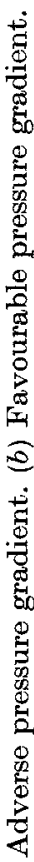

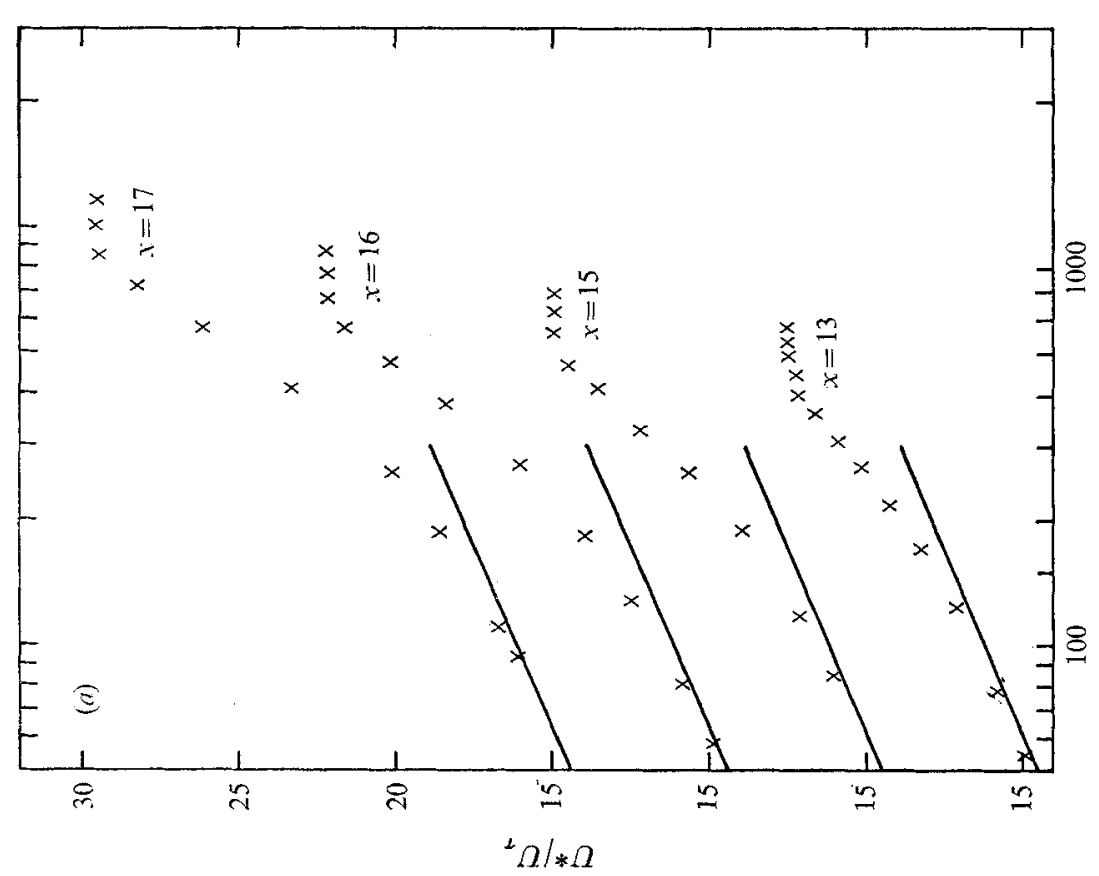

is 

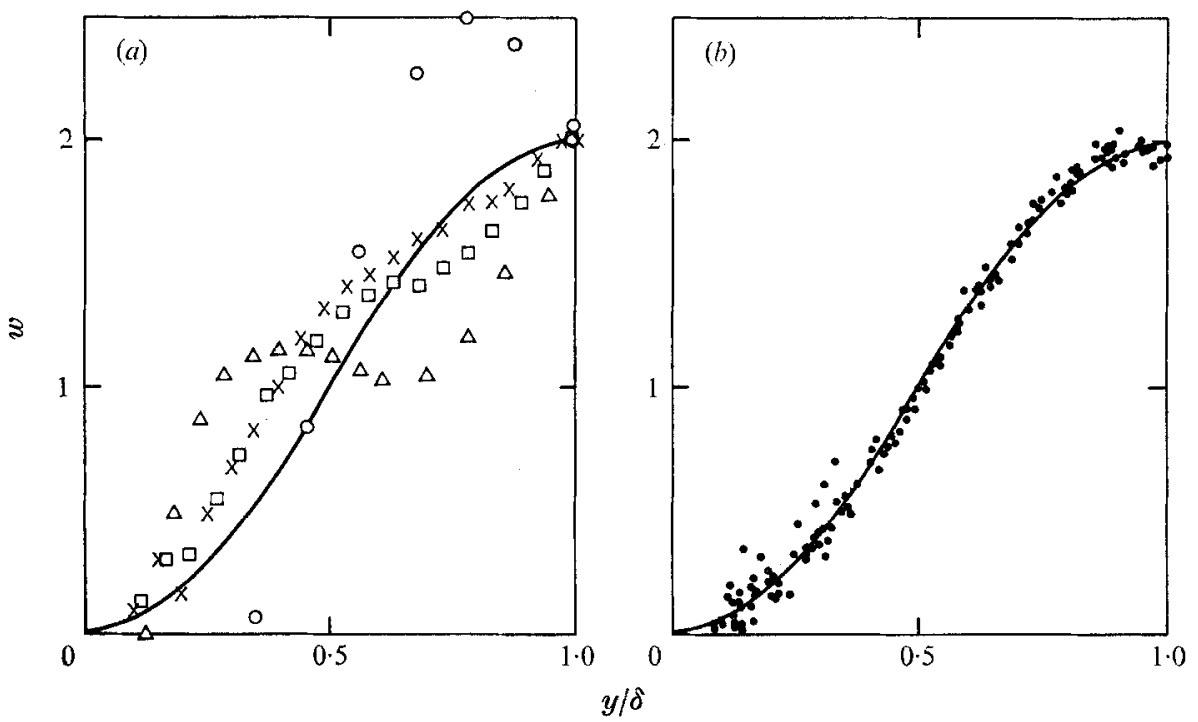

Figure 8. Coles's wake function, $2 \sin ^{2}(\pi y / 2 \delta)$. (a) Favourable pressure gradient: $O$, $x=19 ; \triangle, x=21 \cdot 5 ; \square, x=25.5 ; \times, x=26.5$. (b) Adverse pressure gradient: $x \leqslant 18$.

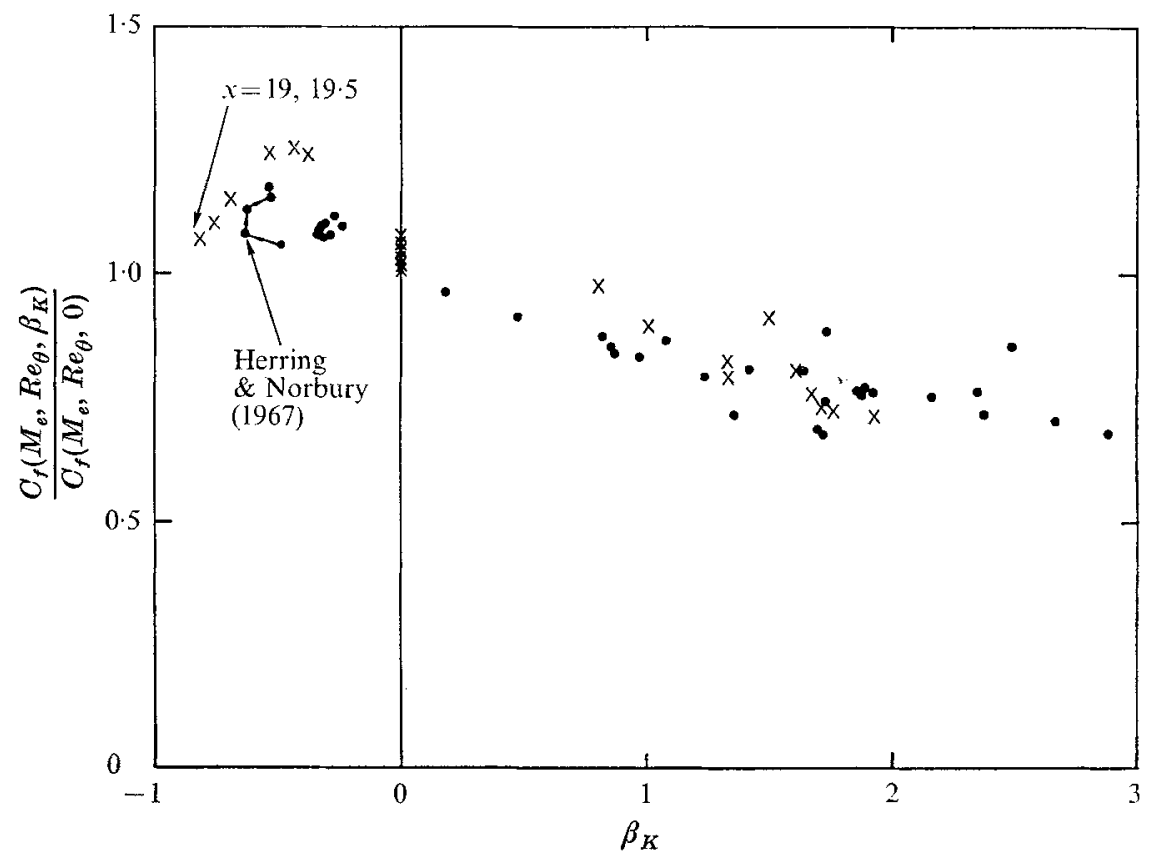

FIGURE 9. Effect of $\beta_{K}$ on skin friction. $\times$, present results;

๑, low-speed data from Coles \& Hirst (1969). 
Similar remarks apply to the correlation of Clauser's integral shape parameter $G$, defined as

$$
\begin{aligned}
& G \equiv \int_{0}^{\delta}\left(\frac{U_{e}^{*}-U^{*}}{U_{\tau}}\right)^{2} d(y / \Delta), \\
& \Delta \equiv \int_{0}^{\delta} \frac{U_{e}^{*}-U^{*}}{U_{\tau}} d y,
\end{aligned}
$$

as is shown in figure 11. In fact, both the low- and high-speed data show fair agreement with the low-speed theoretical result of Mellor \& Gibson (1966) indicated by the solid line.

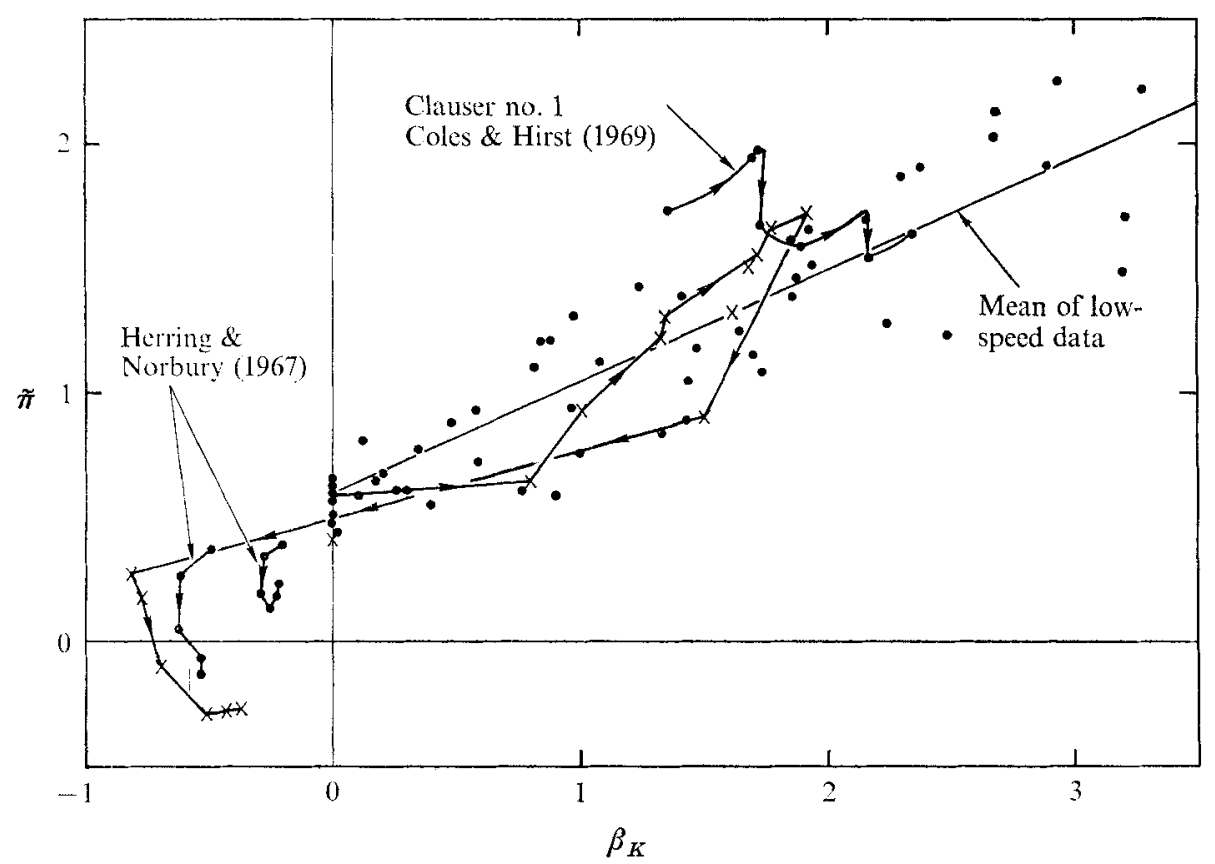

FIGURE 10. Wake strength parameter $\tilde{\pi}$ correlated with $\beta_{K}$. $x$, present results;, low-speed data, Coles \& Hirst (1969).

There are two important conclusions to be drawn at this point based on the previous discussions. The first is that the boundary layer is in approximate local equilibrium throughout the adverse pressure gradient region. By local equilibrium it is meant that the entire boundary-layer profile is characterized by local conditions only (i.e. $C_{f}, \beta_{K}, M_{e}, \delta$ and $T_{w} / T_{e}$ ). The upstream history of the boundary layer and the fact that $\beta_{K}$ is not constant do not have an appreciable effect. The second conclusion is that correspondence between high-speed and low-speed flows has been established based on the pressure gradient parameter $\beta_{K}$.

In order to demonstrate both the above conclusions somewhat more clearly, figure 12 shows the velocity profiles plotted in Clauser's defect form

$$
\left(U_{e}^{*}-U^{*}\right) / U_{\tau}=F\left(y / \Delta ; \beta_{K}\right)
$$


for selected positive values of $\beta_{K}$. Also shown are low-speed data for the same value of $\beta_{K}$. The excellent correspondence between the high-speed and low-speed profiles is evident.

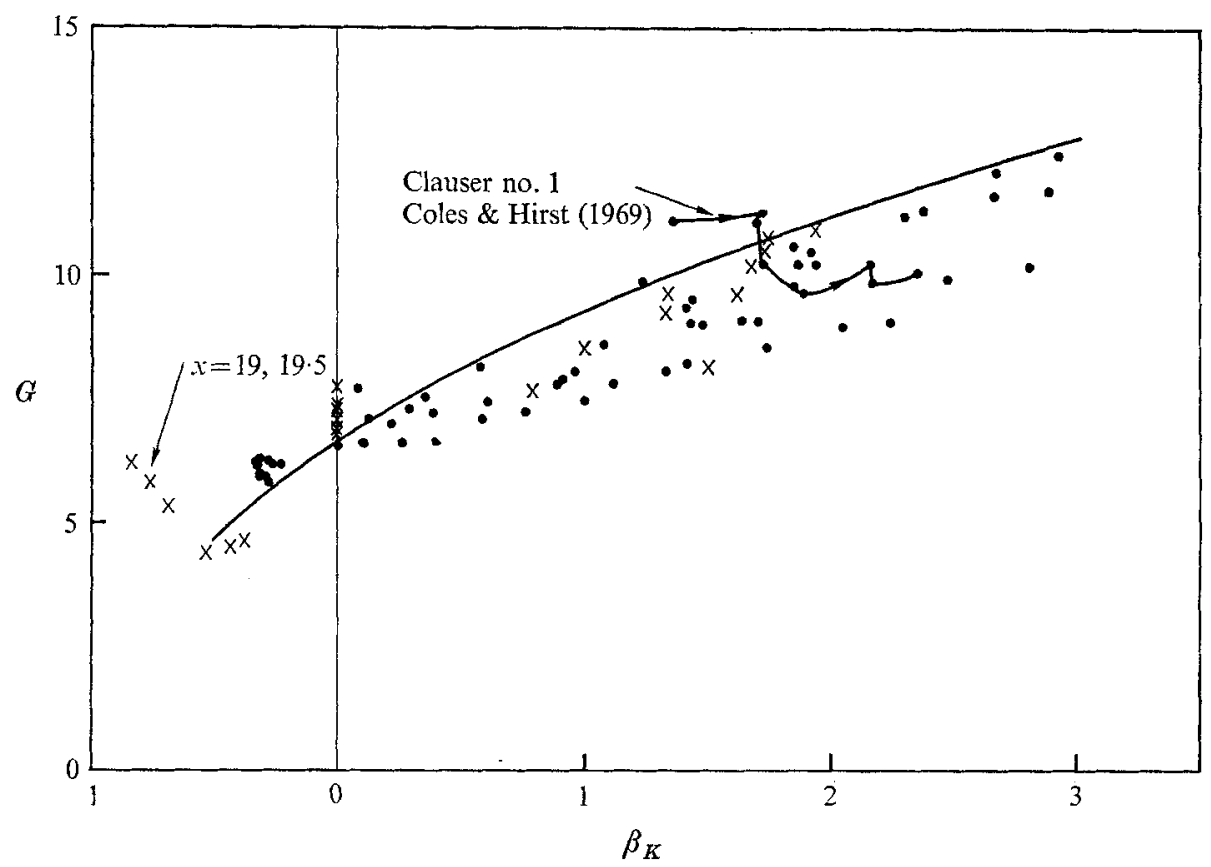

Figure 11. Integral parameter $G$, correlation with $\beta_{K^{*}} \times$, present results; , low-speed data, Coles \& Hirst (1969); —_, Mellor \& Gibson (1966), theory.

\subsection{Favourable pressure gradient}

The adverse pressure gradient data have been shown to be well represented by a local equilibrium concept. The results for the favourable pressure gradient are more accurately described as a relaxation to an equilibrium state. As was shown in figure 2 , the boundary layer was subjected to a strong negative pressure gradient beginning at $x=18.5 \mathrm{in}$. The resulting boundary-layer development shows a number of interesting overall features. While the boundary-layer thickness increased by nearly a factor of 5 over a distance of $8 \mathrm{in} ., R e_{\theta}$ was almost invariant. Further, a check on the mass balance shows that the edge of the boundary layer is approximately a streamline, which is a result of a marked reduction in the turbulent entrainment $\uparrow$.

The velocity profiles are characterized by an extended law-of-the-wall region and a virtual invariance of thickness in terms of the wall co-ordinate $U_{\tau} \delta / \nu_{w}$. The $\beta_{K}=-0.5$ experiment of Herring \& Norbury (1967) shows a similar behaviour with the fortuitous result that $U_{\tau} \delta / \nu_{w} \simeq 2500$ characterizes both experiments. By far the largest values of $\left|d \beta_{K}\right| d x \mid$ occur at the beginning of the

$\dagger$ It is speculated that this is largely a result of the effect of the strong expansion on intermittency, which in turn has a key role in the entrainment process. Unfortunately, no hot-wire measurements were made with which to substantiate this speculation. 

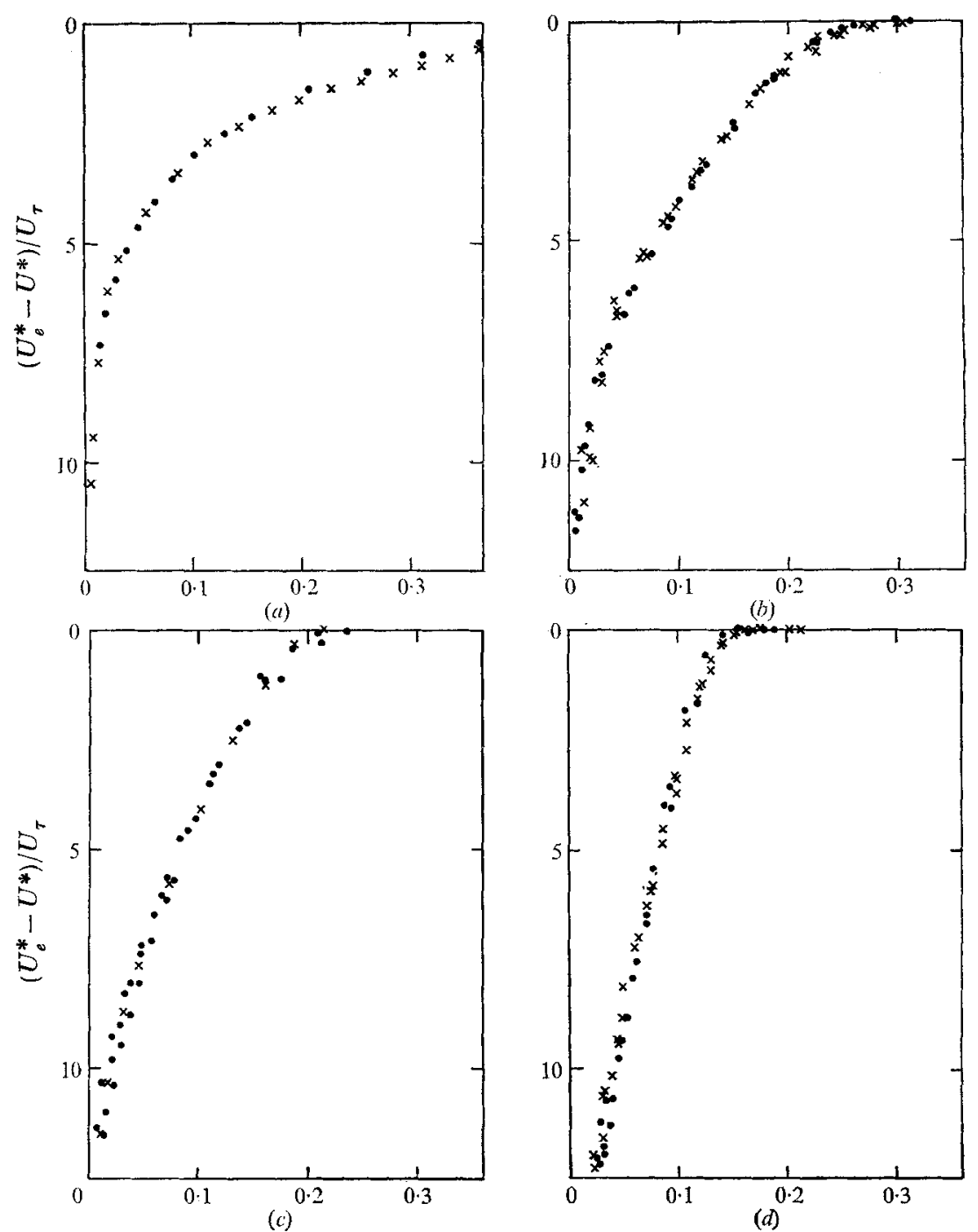

$y / \Delta$

Figure 12. Van Driest velocity profile-Clauser defect form. $(a) \beta_{K}=-0.5 ;(b), \beta_{K}=0$; (c) $\beta_{K}=1 \cdot 0 ;$ (d) $\beta_{K}=1 \cdot 7$. $\bullet$, low-speed data, Coles \& Hirst (1969).

expansion where $\beta_{K}$ drops from +1.5 at $x=18 \mathrm{in}$. to -0.8 at $x=19 \mathrm{in}$. Hence it is not surprising that the boundary-layer profiles in this region show evidence of not being in equilibrium. This can be seen in figure $8(b)$, where the data show significant deviations from the classical wake function $\dagger$.

$\dagger$ There is not sufficient experimental evidence (either high-speed or low-speed) to justify the inverted wake function for a negative value of $\tilde{\pi}$. Despite this fact, the values of $U_{T}$ computed using the scheme presented earlier are as good as could be obtained by fitting the logarithmic region alone. However, the indicated values of $\pi$ and $W(y / \delta)$ were computed manually from the data to satisfy the edge boundary condition $W(1)=2$, which showed significant deviations when the 'best fit' procedure was followed. 
The correlation of $C_{f e}, \tilde{\pi}$ and $G$ with $\beta_{K}$ (figures $4(c), 5(a)$ and 11 ) shows the non-equilibrium behaviour of both the present results and the low-speed data. In both cases the boundary-layer development was observed over a distance of approximately 50 initial boundary-layer thicknesses, and the final profile appeared close to an asymptotic state. The final velocity profile of each experiment is shown in Clauser's defect form in figure $12(d)$, where the agreement is as good as previous comparisons with adverse pressure gradient data.

\section{Conclusions}

For an adiabatic wall, supersonic Mach number $M_{e} \leqslant 4$, and for mild adverse pressure gradients, $\beta_{K} \leqslant 2$, the turbulent boundary-layer velocity profiles have shown to be in good agreement with classical low-speed results when viewed in the co-ordinates suggested by Van Driest. For the pressure distribution investigated, all profiles in regions of adverse pressure gradient appear to be in local equilibrium, i.e. characterized by $\beta_{K}$ and not strongly dependent on their history. The boundary layer, when subjected to a large negative $d \beta_{K} / d x$, was shown to be poorly characterized by the law of the wake but was found to relax to an equilibrium-like profile after 50 initial boundary-layer thicknesses. A similar result is noted for the low-speed constant $\beta \simeq-0.5$ experiment of Herring \& Norbury. The final profile of their experiment and the present experiment show good agreement in Van Driest co-ordinates.

The authors gratefully acknowledge the support of the Advanced Research Projects Agency and the U.S. Air Force Space and Missile Systems Organization under Contract FO4701-71-C-0040 and the co-operation of the staff of the von Kármán Gas Dynamics Facility at AEDC where the experiments were performed.

\section{REFERENCES}

Abarbanel, S. S., Hakkinen, R. J. \& Trilling, L. 1959 Use of a Stanton tube for skinfriction measurements. N.A.S.A. Memo. 2-17-59W.

Alber, I. E. \& Coats, D. E. 1969 Analytical Investigation of Equilibrium and NonEquilibrium Compressible Turbulent Boundary Layers. A.I.A.A. Paper, 69-689.

BARonTI, P. O. \& LIBBY, P. A. 1966 Velocity profiles in turbulent compressible boundary layers. A.I.A.A. J. 4, 193-201.

Bradshaw, P. \& Ferriss, D. H. 1965 The response of a retarded equilibrium turbulent boundary layer to the sudden removal of pressure gradient. Nat. Phys. Lab. Aero. Rep. 1145.

Bradshaw, P. \& Gregory, M. A. 1961 The determination of local turbulent skin friction from observations in the viscous sublayer. Aero. Res. Counc. R.\& M. 3202.

BRoTT, D. X., YANTA, W. J. \& LEE, R. E. 1968 An experimental investigation of the surface Pitot probe including effects of heat transfer and compressibility. Nav. Ord. Lab. Tech. Rep. 68-151.

Claudser, F. H. 1956 The turbulent boundary layer. Advanc. Appl. Mech. 4, 1-51.

Coles, D. E. 1953 Measurements in the boundary layer on a smooth flat plate in supersonic flow, 1. The problem of the turbulent boundary layer. Jet Propulsion Lab. Rep. $20-69$. 
Coles, D. E. 1956 The law of the wake in the turbulent boundary layer. J. Fluid Mech. 1, 191-226.

Coles, D. E. 1962 The turbulent boundary layer in a compressible fluid. Rand. Corporation, Santa Monica, California, Rep. R-403-Pr.

Coles, D. E. \& Hirst, E. A. 1969 Proceedings Computation of Turbulent Boundary Layers, 1968 AF'OSR-IF'P-Stanford Conference, vol. II. Thermosciences Division, Stanford University, California.

Herring, H. J. \& Norbury, J. F. 1967 Some experiments on equilibrium turbulent boundary layers in favourable pressure gradients. J. Fluid Mech. 27, 541-549.

HooL, J. N. 1956 Measurements of skin friction using surface tubes. J. Aircraft Eng. 28, $52-53$.

Horkins, E. J. \& INOUYE, M. 1971 An evaluation of theories for predicting turbulent skin friction and heat transfer on flat plates at supersonic and hypersonic Mach numbers, A.I.A.A. J. 9, 993-1003.

Hopkins, E. J. \& KeEner, E. R. 1966 Study of surface Pitots for measuring turbulent skin friction at supersonic Mach numbers-adiabatic wall. N.A.S.A. Tech. Note D-3478.

LEWIS, J. E., KuBota, T. \& WeBb, W. H. 1970 Transformation theory for the adiabatic compressible turbulent boundary layer with pressure gradient. A.I.A.A. J. 8, 16441650.

Matse, G. \& MoDonald, H. 1967 Mixing Length and Kinematic Eddy Viscosity in a Compressible Boundary Layer. A.I.A.A. 5th Aerospace Sciences Meeting.

Mellor, G. L. \& Gibson, D. M. 1966 Equilibrium turbulent boundary layers. J. Fluid Mech. 24, 225-253.

Smith, K. G., Gaudet, L. \& Winter, K. G. 1964 The use of surface Pitot tubes as skin-friction meters at supersonic speeds. Aeron. Res. Counc. R. \& M.3351.

STRATFORD, B. S. 1959 An experimental flow with zero skin friction throughout its region of pressure rise. J. Fluid Mech. 5, 17-35.

VAN DRIEST, E. R. (1951). The turbulent boundary layer in compressible fluids. J. Aero. Sci. 18, 145-160. 


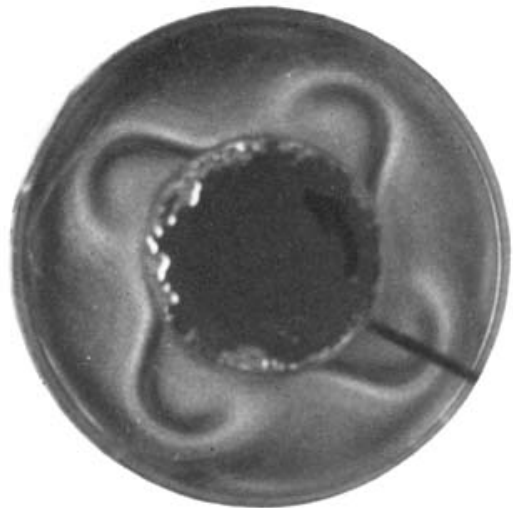

(a)

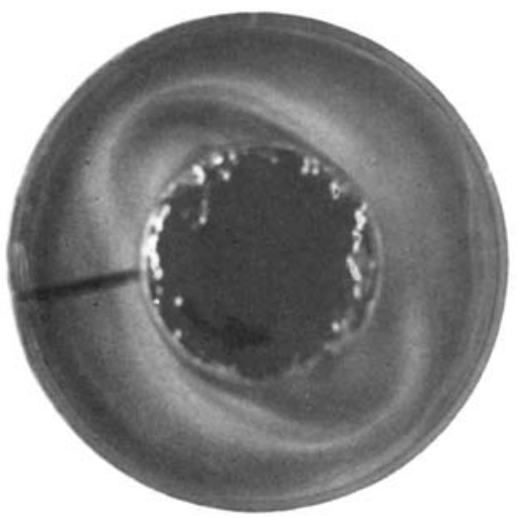

(c)

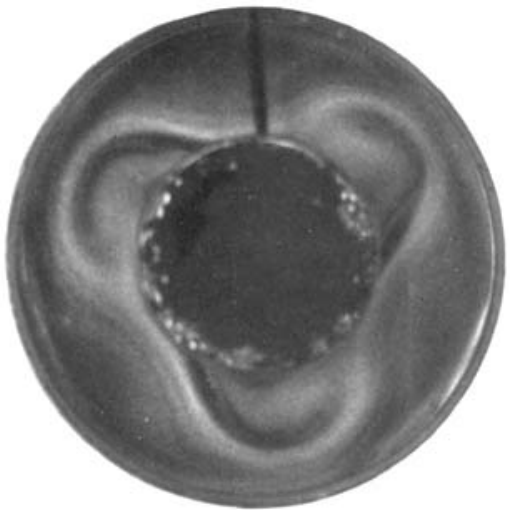

(b)

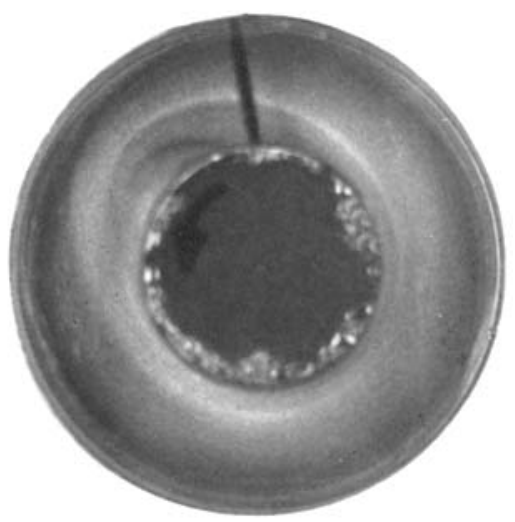

(d)

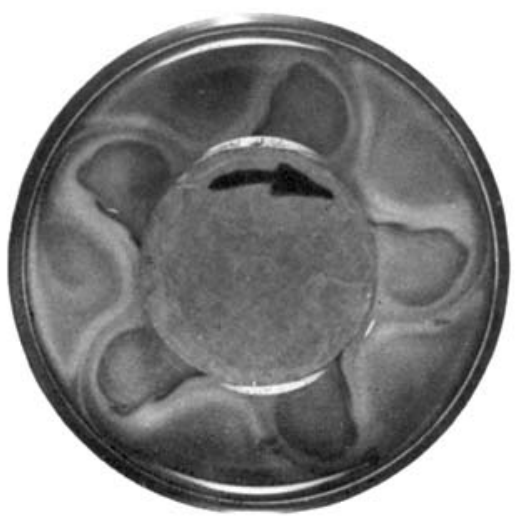

(e)

Figure 3. Some steady-state patterns with a positive temperature gradient and $\Omega=2 \cdot 5 \mathrm{rad} / \mathrm{s}$ for $(a),(b),(c)$ and $(d)$. (a) Four-vortex pattern, $\Delta T_{r}=14 \cdot 6^{\circ} \mathrm{C} ;(b)$ three-vortex pattern, $\Delta T_{r}=17 \cdot 1^{\circ} \mathrm{C} ;(c)$ two-vortex pattern, $\Delta T_{r}=22.0^{\circ} \mathrm{C} ;(d)$ one-vortex pattern, $\Delta T_{r}=30 \cdot 7^{\circ} \mathrm{C}$. (e) Five-vortex pattern under an air surface, $\Omega=3 \cdot 14 \mathrm{rad} / \mathrm{s}, \Delta T_{r}=9 \cdot 4^{\circ} \mathrm{C}$. 


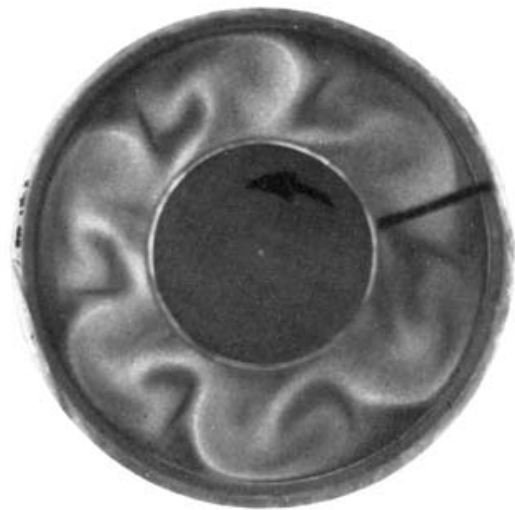

(a)

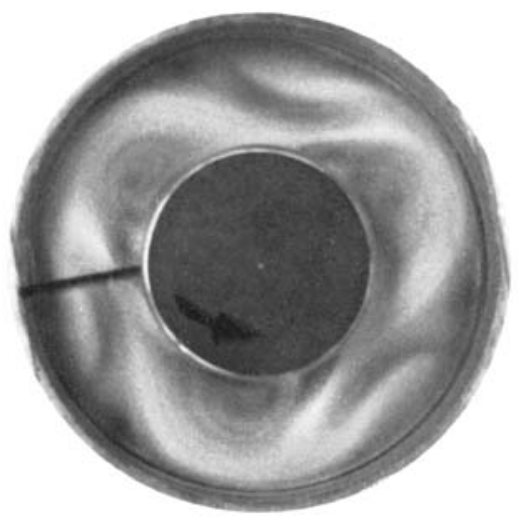

(c)

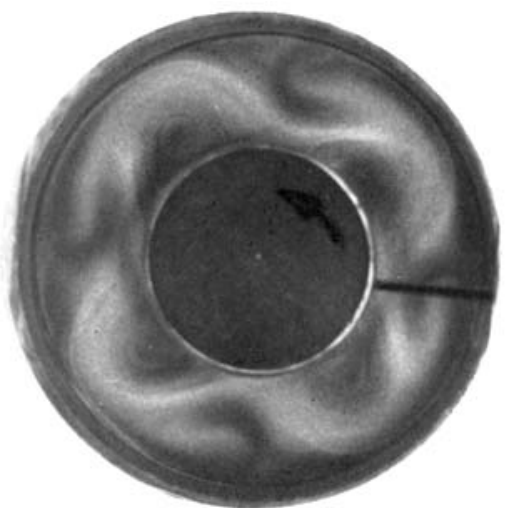

(b)

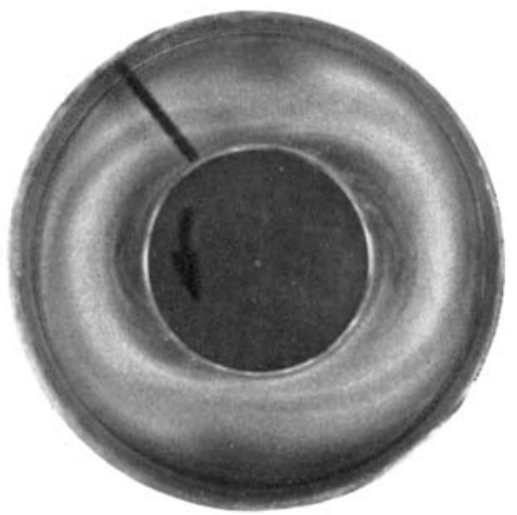

(d)

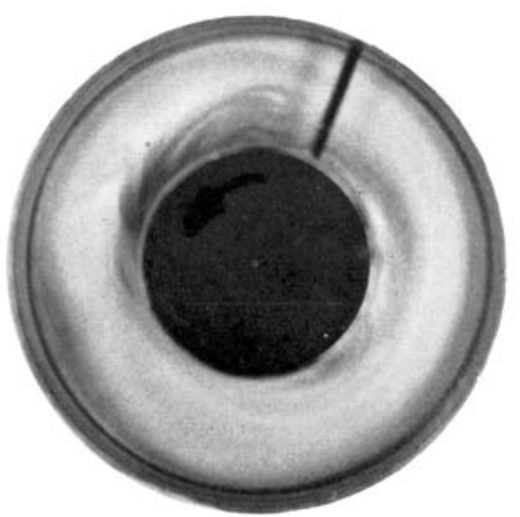

(e)

Figure 4. Some steady-state patterns with a negative temperature gradient and $\Omega=\mathbf{2 \cdot 5}$ $\mathrm{rad} / \mathrm{s}$. (a) Six-vortex pattern, $\Delta T_{r}=-1.8^{\circ} \mathrm{C} ;(b)$ four-vortex pattern, $\Delta T_{r}=-12^{\circ} \mathrm{C}$; (c) three-vortex pattern, $\Delta T_{r}=-20^{\circ} \mathrm{C} ;(d)$ two-vortex pattern, $\Delta T_{r}=-38^{\circ} \mathrm{C} ;(e)$ onevortex pattern, $\Delta T_{r}=-40^{\circ} \mathrm{C}$.

\section{KOSCHMIEDER}




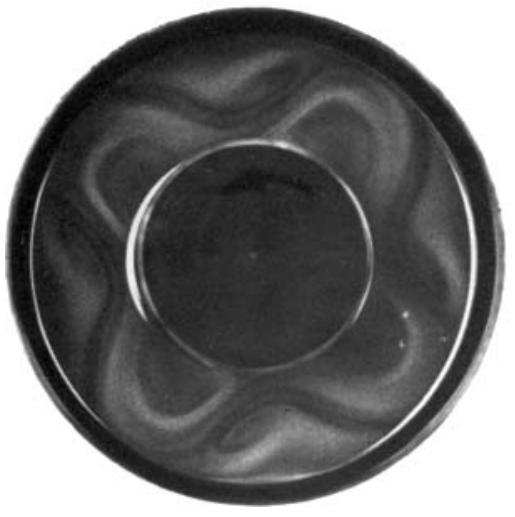

(a)

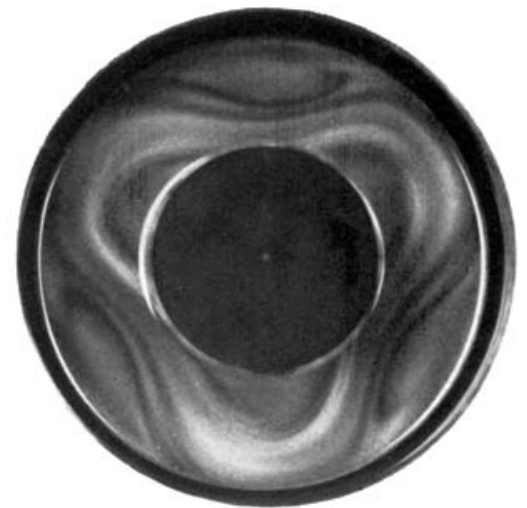

(b)

FIGURE 5. Two steady-state pattorns under an air-surface with a negativo temporature gradient and $\Omega=2.5 \mathrm{rad} / \mathrm{s}$. (a) Four-rortex pattern, $\Delta T_{r}=-15^{\circ} \mathrm{C}$; (b) threo-vortex pattern, $\Delta T_{r}=-20^{\circ} \mathrm{C}$.

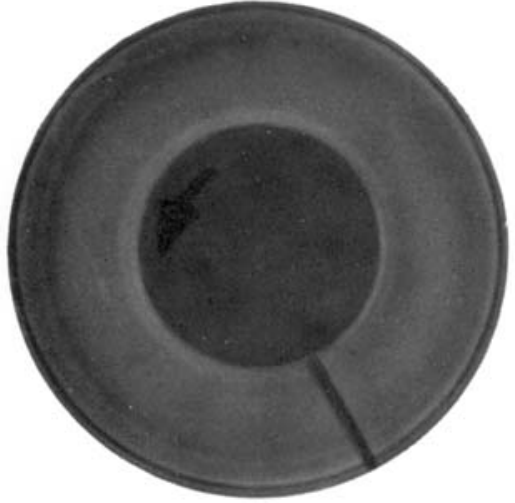

(a)

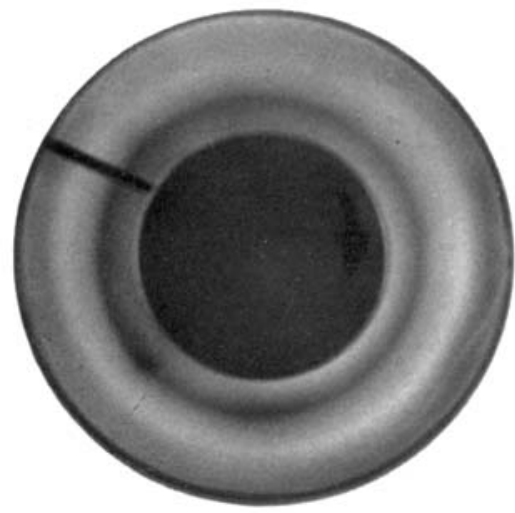

(c)

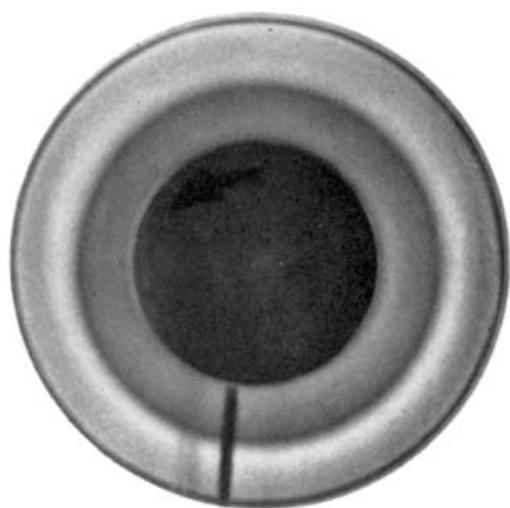

(b)

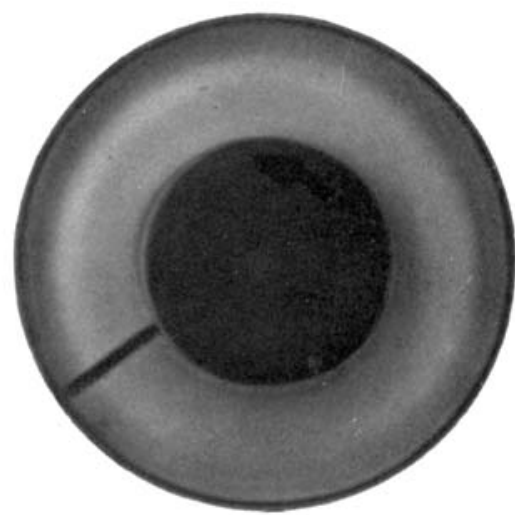

(d)

FIGURE 7. Transition to flow in the upper symmetrical regime after the sudden application of a positive temperature gradient. $\Omega=0.7 \mathrm{rad} / \mathrm{s}, \Delta T= \pm 4.5^{\circ} \mathrm{C}$. (a) Warm cell at outer cylinder, cold cell at inner eylindor, $t=12 \mathrm{~s}$; $(b)$ warm-cell and cold coll at $t=24 \mathrm{~s}$; (c) expansion of tho warm cell, $t=31 \mathrm{~s} ;(d)$ symmetric flow, $t=37 \mathrm{~s}$.

\section{KOSCHMIEDER}




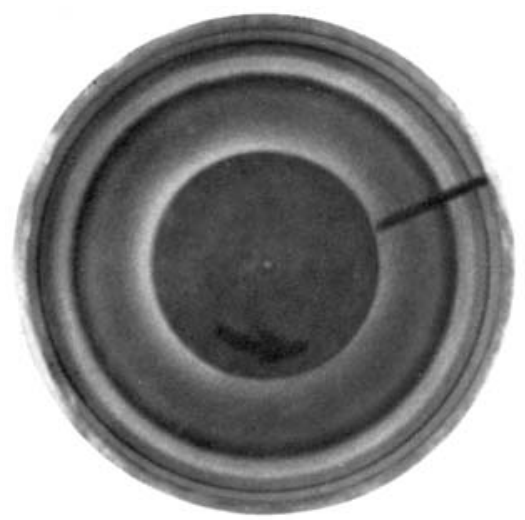

(a)

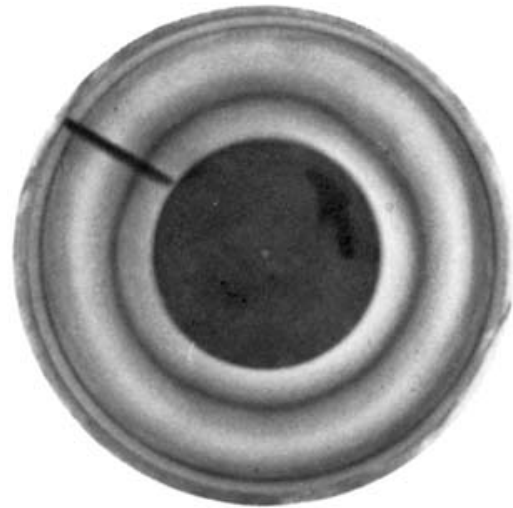

(c)

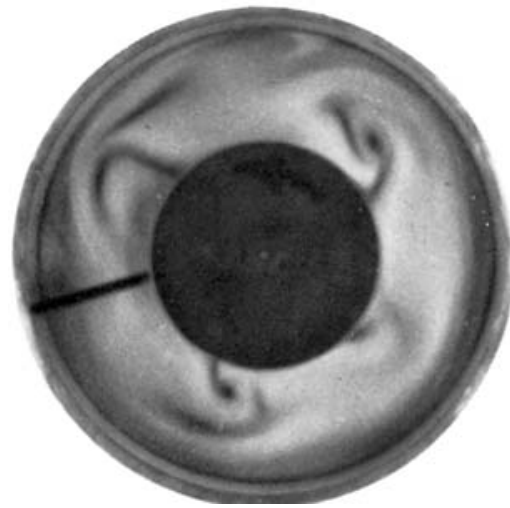

(e)

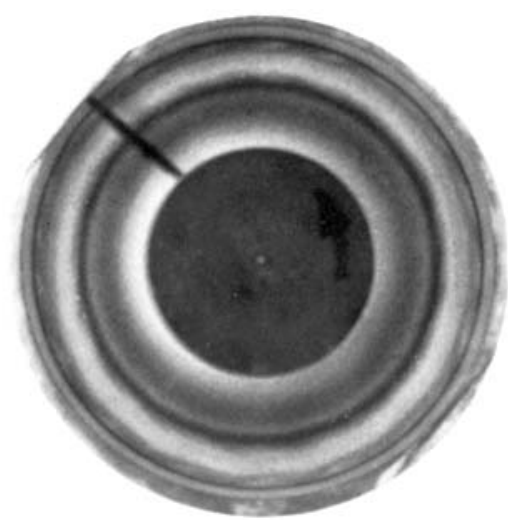

(b)

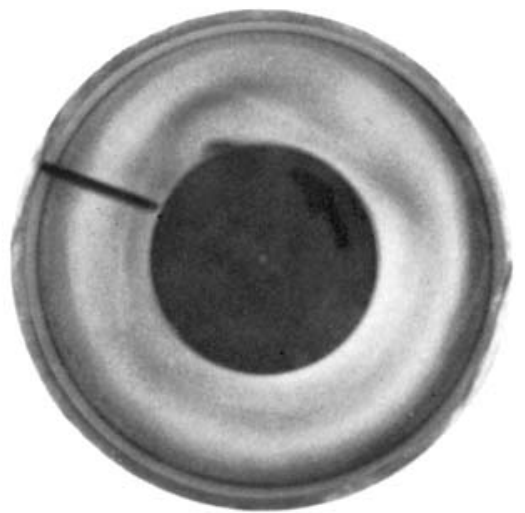

(d)

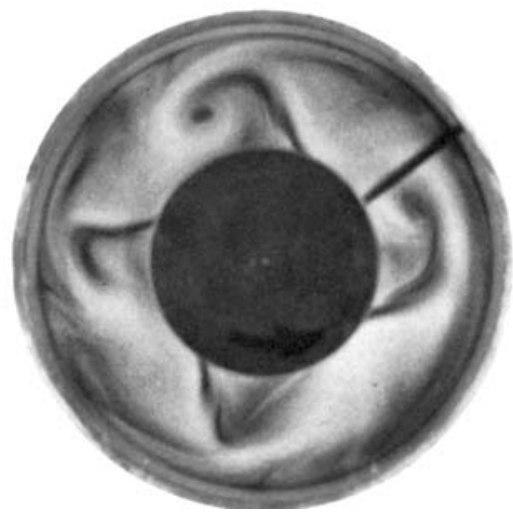

(f)

Figure 9. Development of vortices after the sudden application of a positive temperature gradient. $\Omega=2.5 \mathrm{rad} / \mathrm{s}, \Delta T= \pm 5^{\circ} \mathrm{C}$. (a) Warm cell, weak counter cell and cold cell, $t=21 \mathrm{~s}$; (b) shrinking counter cell, $t=29 \mathrm{~s} ;(c)$ warm cell overruns counter cell, $t=57 \mathrm{~s} ;(d)$ warm cell at inner cylinder, $t=73 \mathrm{~s} ;(e)$ four-vortices form, $t=89 \mathrm{~s} ;(f)$ four-vortices at $t=120 \mathrm{~s}$.

\section{KOSCHMIEDER}




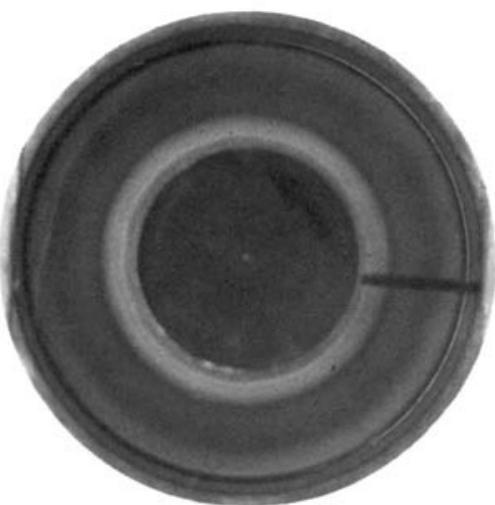

(a)

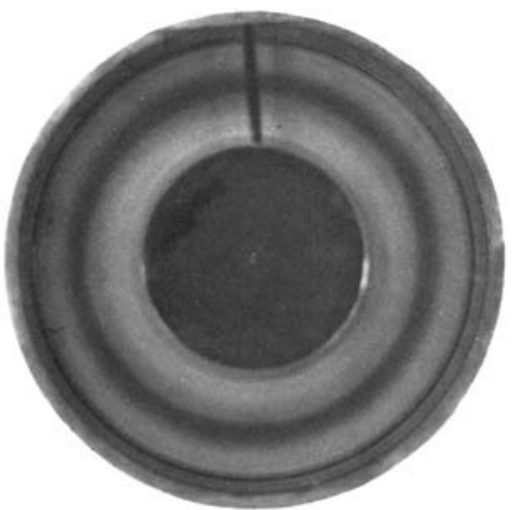

$(c)$

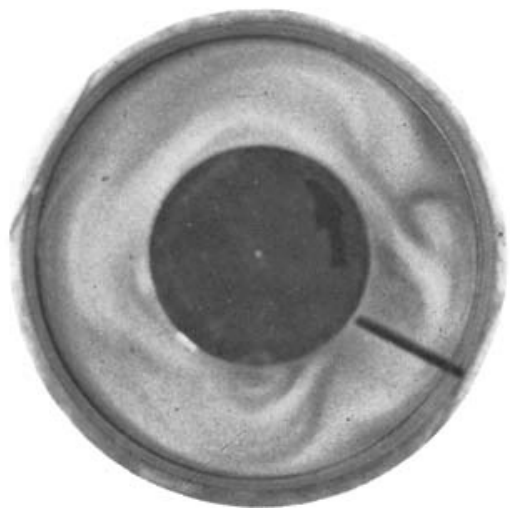

(e)

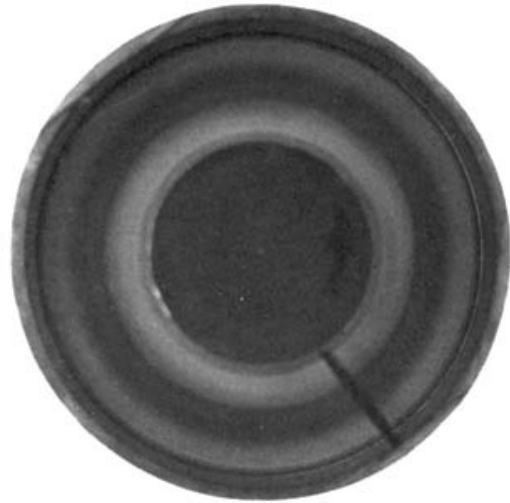

(b)

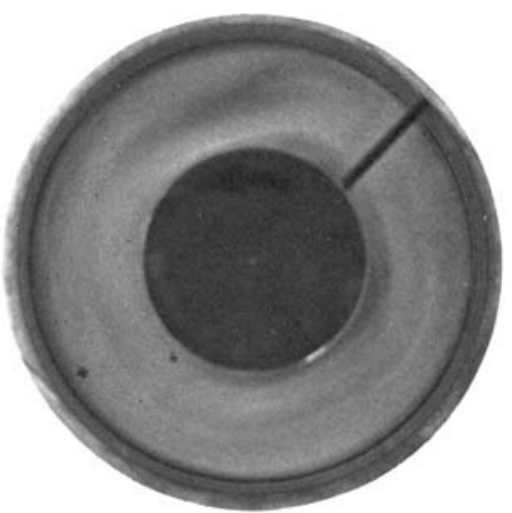

$(d)$

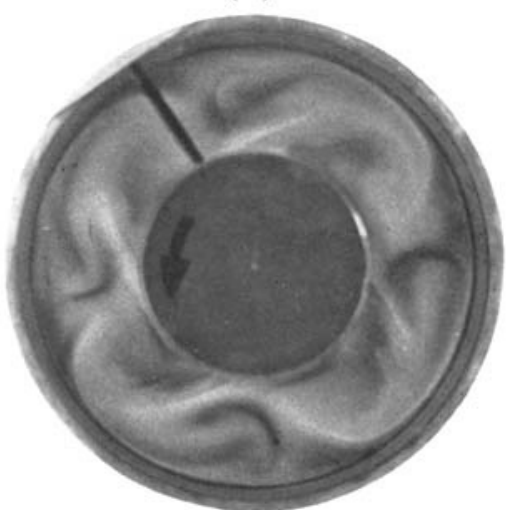

$(f)$

Figure 11. Development of vortices after the sudden application of a negative temperature gradient. $\Omega=2.5 \mathrm{rad} / \mathrm{s}, \Delta T=\mp 5^{\circ} \mathrm{C}$. (a) Warm cell, counter coll and cold cell, $t=23 \mathrm{~s}$; (b) shrinking counter cell, $t=32 \mathrm{~s}$; (c) warm cell overruns counter cell, $t=46 \mathrm{~s}$; $(d)$ warm cell at outor cylinder, $t=83 \mathrm{~s} ;(e)$ vortices form $t=93 \mathrm{~s} ;(f)$ four vortices at $t=150 \mathrm{~s}$.

\section{KOSCHMIEDER}




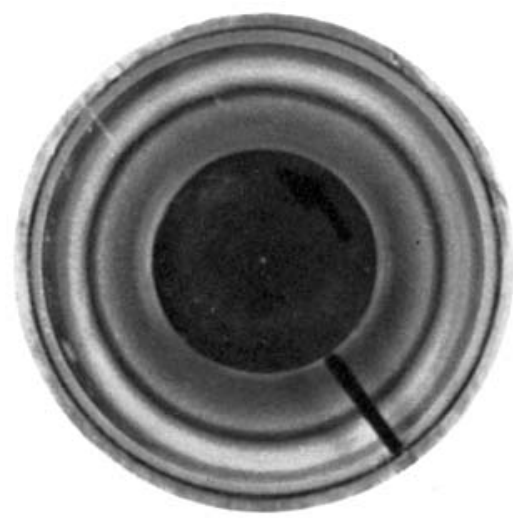

(a)

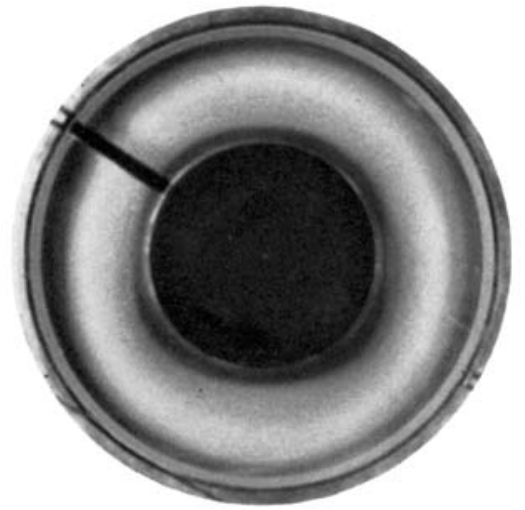

(c)

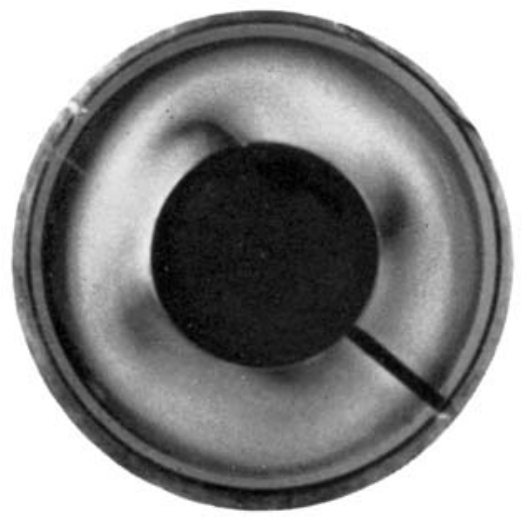

(e)

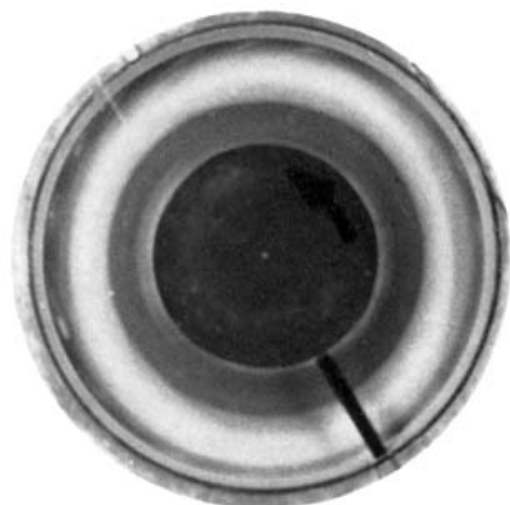

(b)

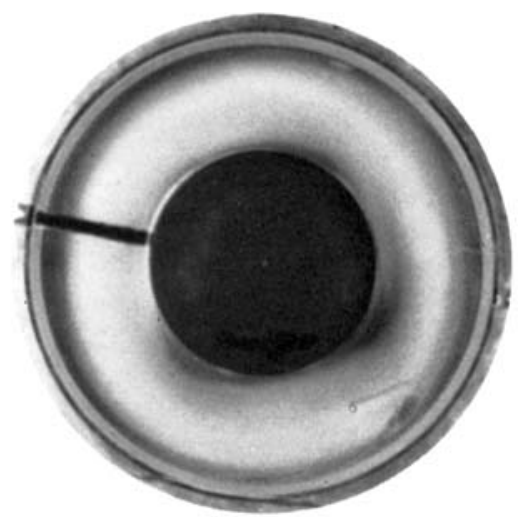

(d)

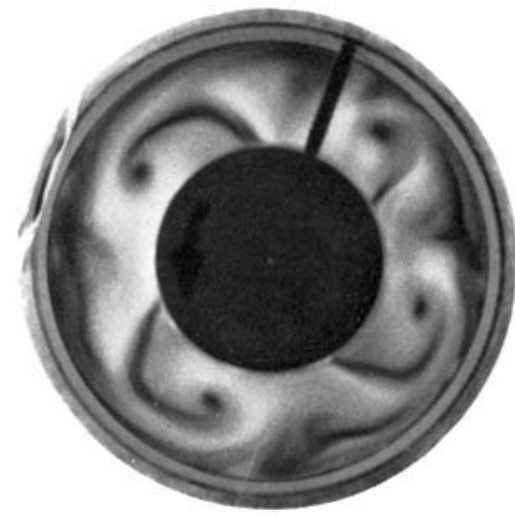

$(f)$

Figure 12. Development of vortices after sudden heating of the outer cylinder. $\Omega=2 \cdot 5 \mathrm{rad} / \mathrm{s}$, $\Delta T_{0}=+10^{\circ} \mathrm{C}$. (a) Warm cell at outer cylinder, $t=32 \mathrm{~s}$; (b) warm cell expands, $t=50 \mathrm{~s}$; (c) warm cell at inner cylinder, $t=77 \mathrm{~s} ;(d)$ vortices form, $t=87 \mathrm{~s}$; $(e)$ vortices form, $t=89 \mathrm{~s}$; $(f)$ four vortices at $t=180 \mathrm{~s}$.

\section{KOSCHMIEDER}




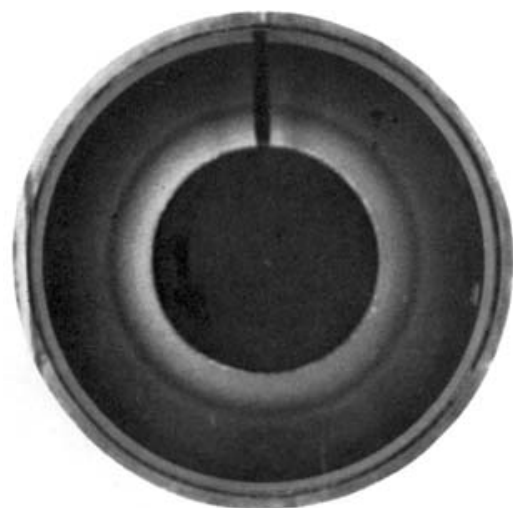

(a)

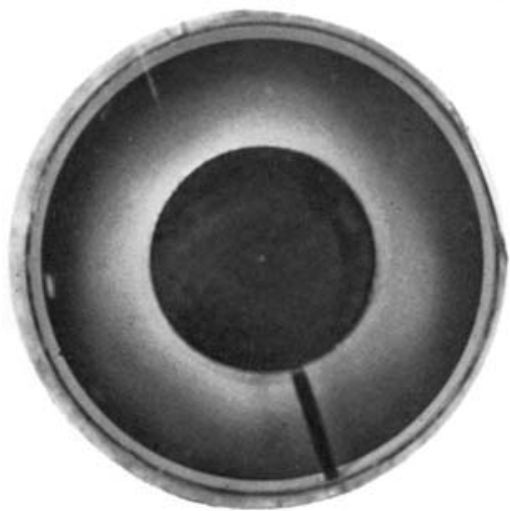

(c)

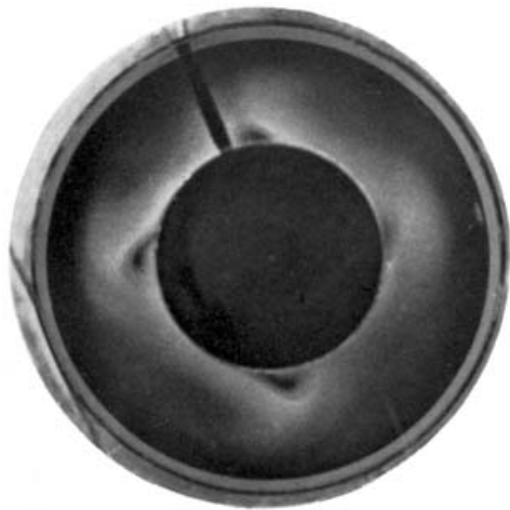

(e)

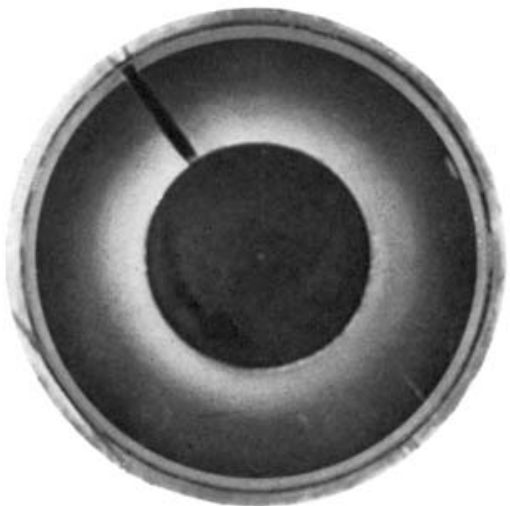

(b)

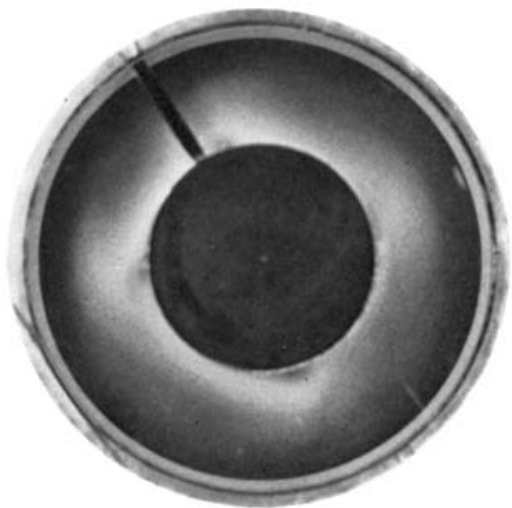

(d)

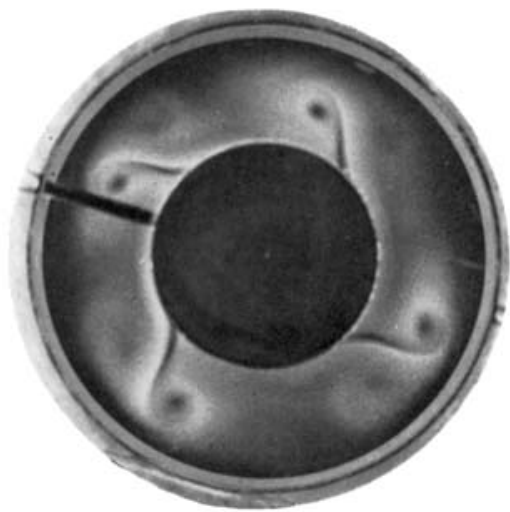

(f)

Figure 13. Development of vortices after sudden cooling of the inner cylinder. $\Omega=2.5 \mathrm{rad} / \mathrm{s}$, $\Delta T_{i}=-10^{\circ} \mathrm{C}$. (a) Cold cell at inner cylinder, $t=51 \mathrm{~s} ;(b)$ cold cell expands, $t=79 \mathrm{~s}$; (c) deformation of cold cell, $t=96 \mathrm{~s} ;(d)$ vortices form, $t=103 \mathrm{~s}$; $(e)$ vortices form, $t=107 \mathrm{~s}$; $(f)$ four vortices, $t=117 \mathrm{~s}$. 

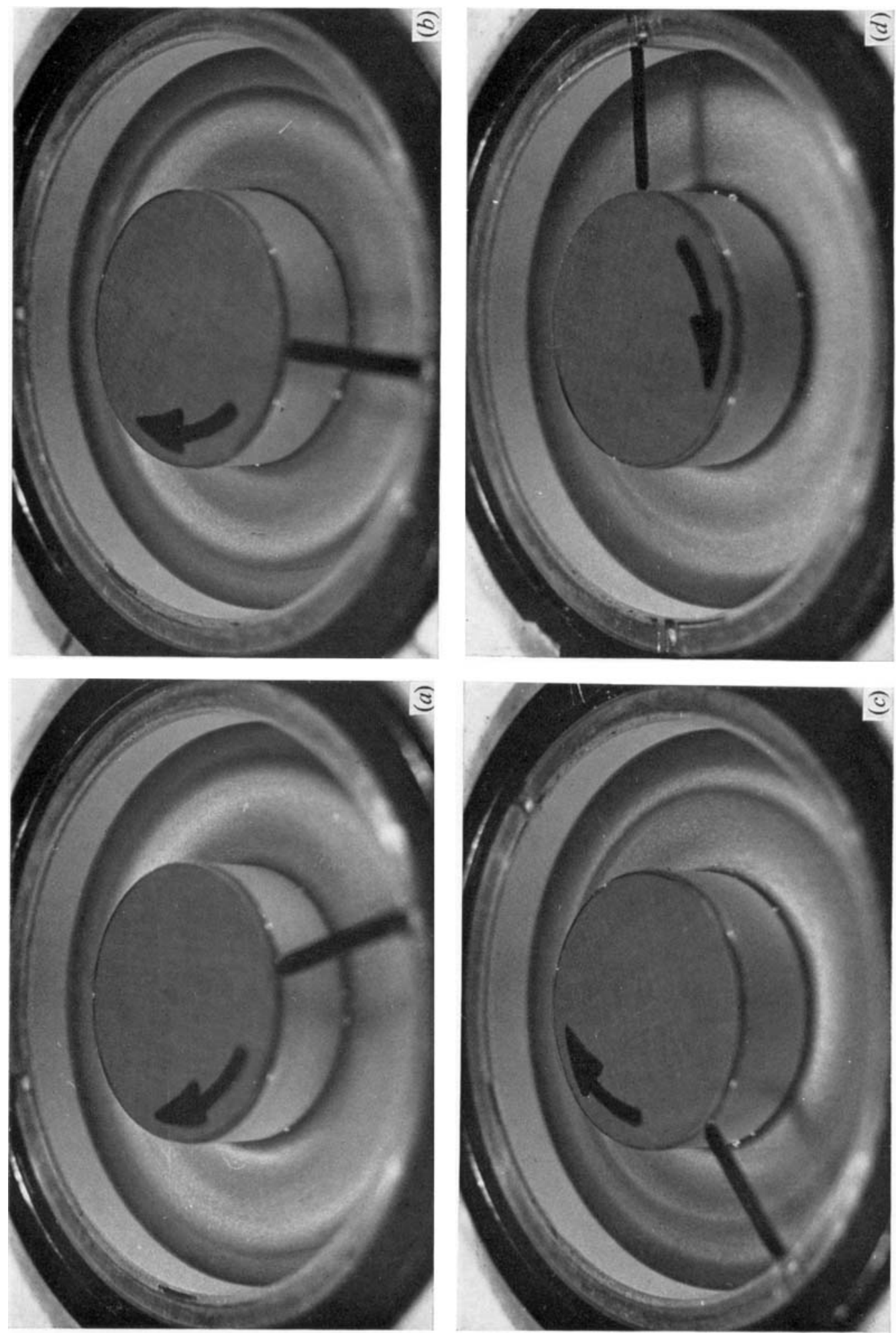

离
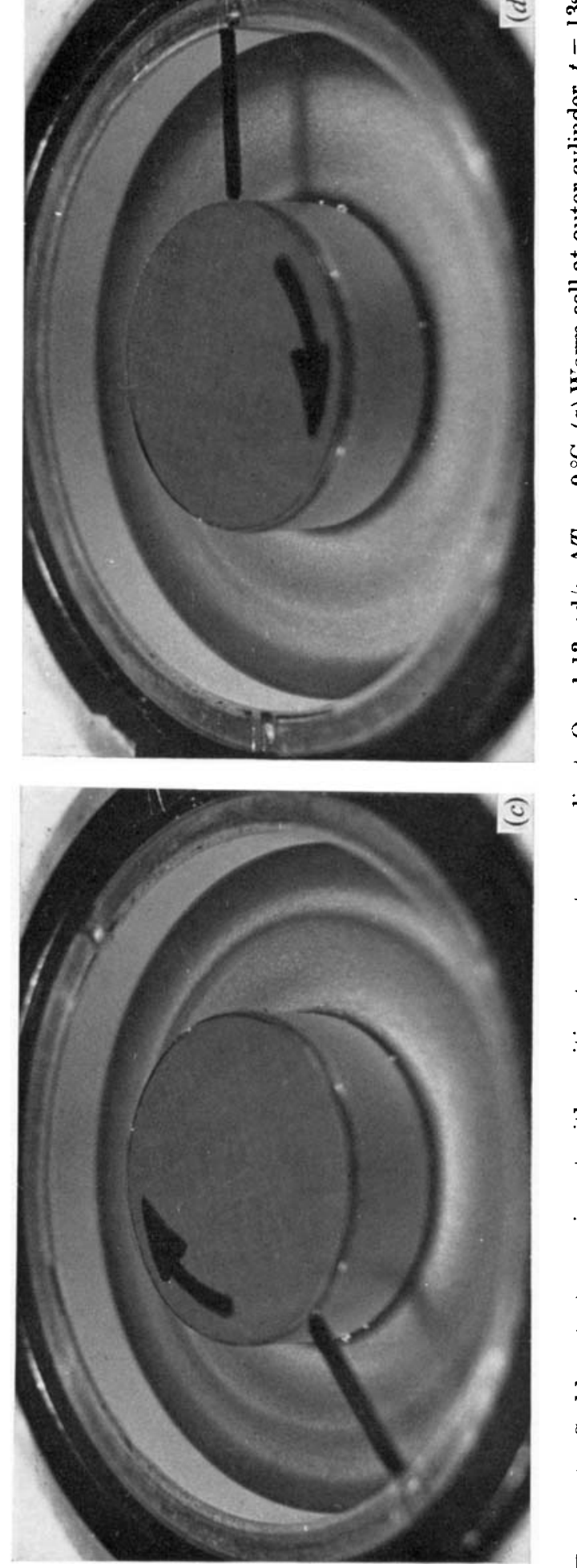

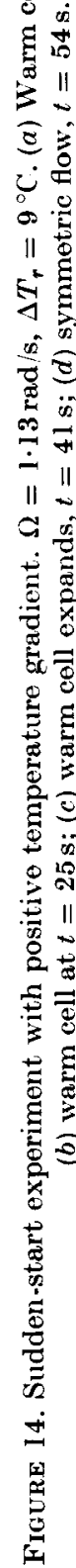




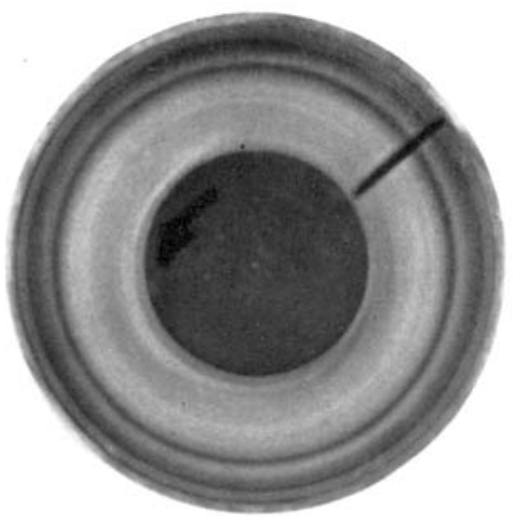

(a)

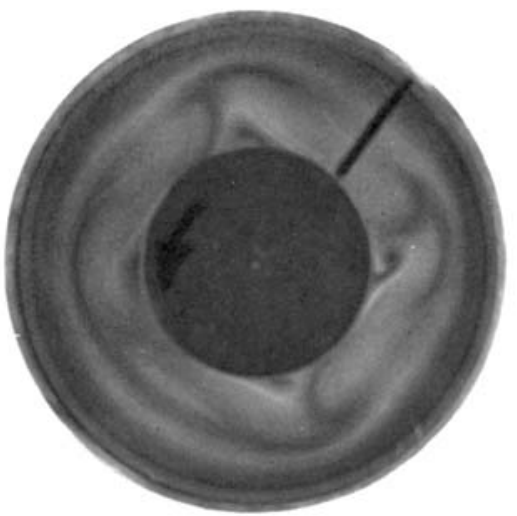

(c)

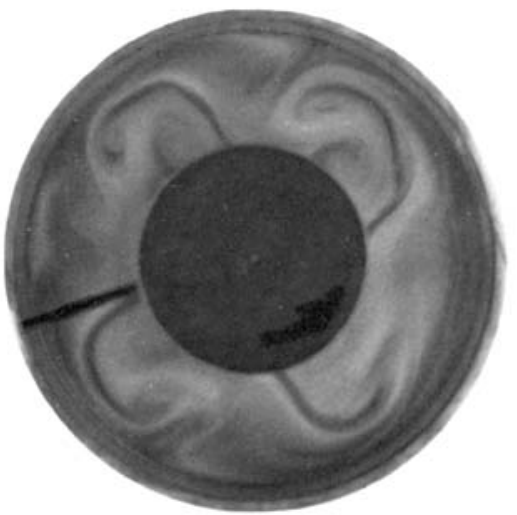

(e)

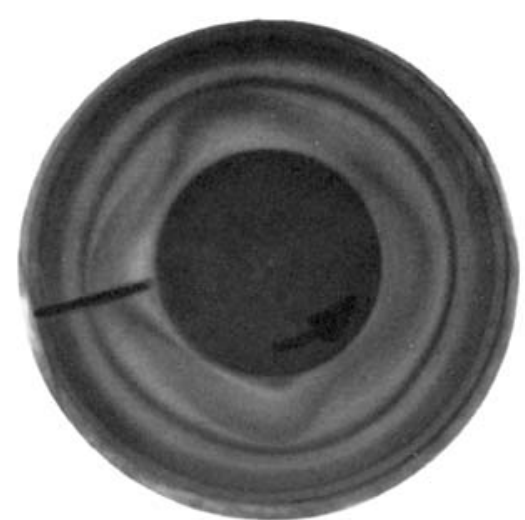

(b)

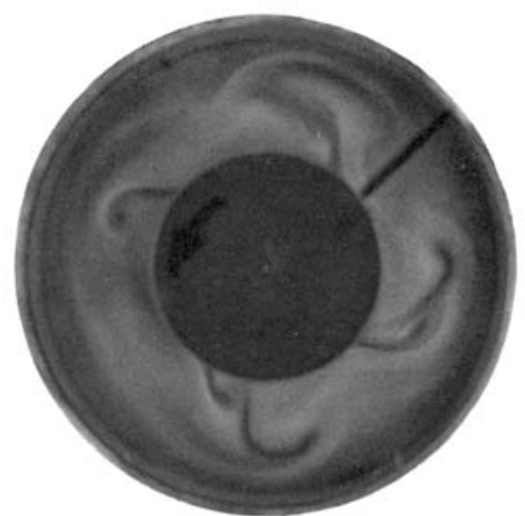

$(d)$

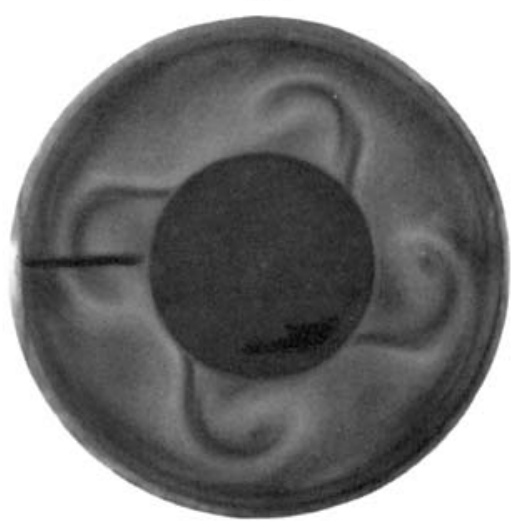

(f)

Figure 15. Development of vortices after a sudden start with a positive temperature gradient. $\Omega=2.5 \mathrm{rad} / \mathrm{s}, \Delta T_{r}=10^{\circ} \mathrm{C}$. (a) Warm cell at outer cylinder, $t=49 \mathrm{~s}$; (b) deformation of symmetric flow, $t=76 \mathrm{~s} ;(c)$ vortices form, $t=86 \mathrm{~s}$; $(d)$ vortices form, $t=91 \mathrm{~s} ;(e)$ four vortices, $t=97 \mathrm{~s} ;(f)$ four vortices, $t=158 \mathrm{~s}$.

\section{KOSCHMIEDER}




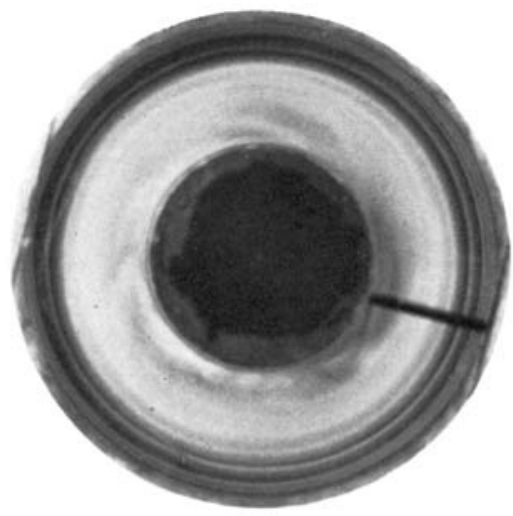

(a)

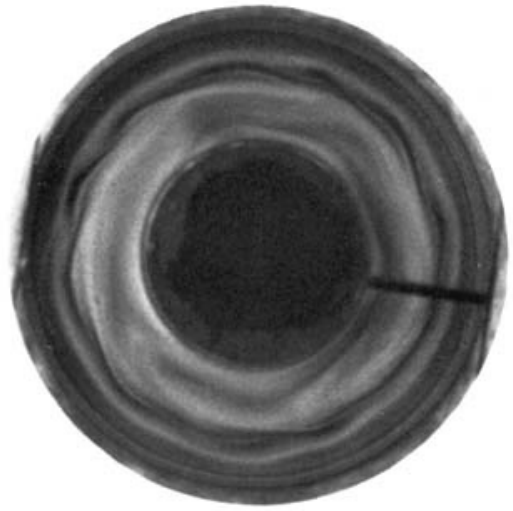

(c)

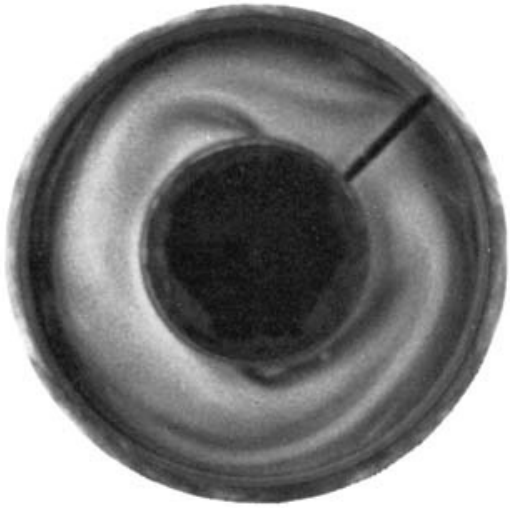

(e)

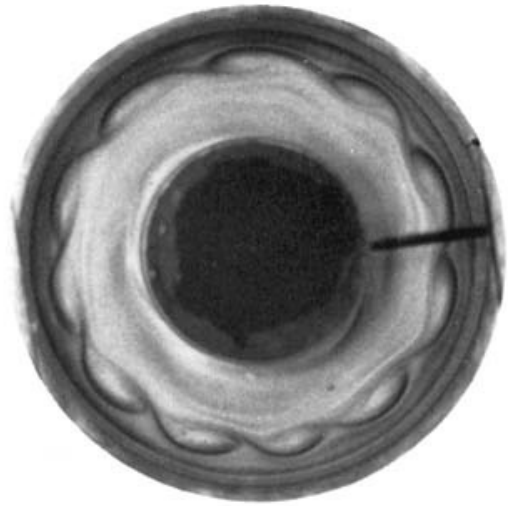

(b)

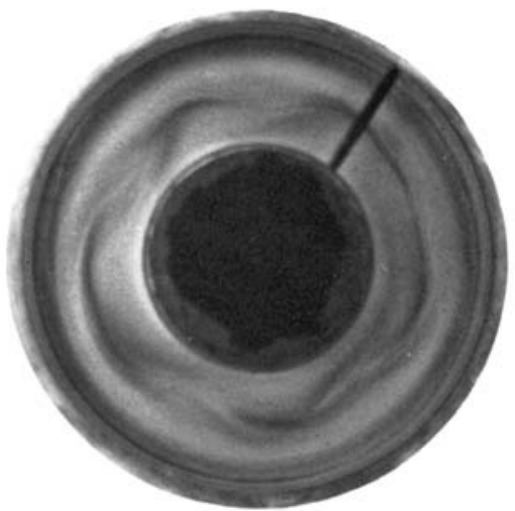

(d)

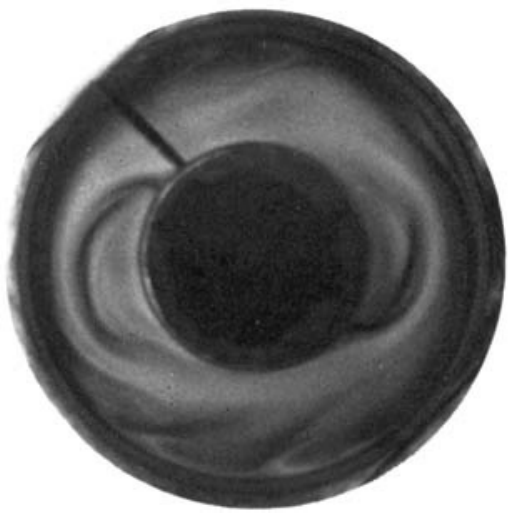

(f)

Figtine 16. Development of vortices after a sudden start with a positive temperature gradient. $\Omega=2.5 \mathrm{rad} / \mathrm{s}, \Delta T_{r}=30^{\circ} \mathrm{C}$. (a) Warm cell at outer cylinder, $t=21 \mathrm{~s} ;(b)$ short waves at $t=29 \mathrm{~s} ;(c)$ flow at $t=35 \mathrm{~s} ;(d)$ transient waves, $t=50 \mathrm{~s} ;(e)$ two vortices appear, $t=68 \mathrm{~s}$; (f) two vortices at $t=86 \mathrm{~s}$.

\section{KOSCHMIEDER}

